Supporting information

\title{
Reactions of in situ Generated N-Boc Nitrones with Aromatic and Heteroaromatics Grignard Reagents: Application to the Synthesis of Zileuton
}


- S3 : Tools, Reagents, Solvents and Procedures

- S4 : Experimental procedures and characterisation data for compounds 1

- S9 : Experimental procedures and characterisation data for compounds 4

- S13 : Experimental procedures and characterisation data for compounds 6

- S16 : Experimental procedures and characterisation data for compound $7 \mathbf{b}$

- S17 : Experimental procedures and characterisation data for compounds 11

- S20 : Experimental procedures and characterisation data for compounds 12

- S23 : ${ }^{1} \mathrm{H}$ and/or ${ }^{13} \mathrm{C}$ NMR spectra of all new compounds 


\section{Tools, Reagents, Solvents and Procedures}

${ }^{1}$ H NMR-Spectra (300 MHz), and ${ }^{13} \mathbf{C}$ NMR-spectra (75.5 MHz) spectra were recorded on an Advance300 spectrometer. Chemical shifts are given in ppm $(\delta)$ and were referenced to the internal solvent signal or to TMS used as an internal standard. Multiplicities are declared as follow: $s$ (singlet), br $s$ (broad singlet), $d$ (doublet), $t$ (triplet), $q$ (quadruplet), $d d$ (doublet of doublet), $d t$ (doublet of triplet), $m$ (multiplet). Coupling constants $J$ are given in $\mathrm{Hz}$.

Infrared spectra (IR) were obtained either as neat films on sodium chloride discs or in potassium bromide plates. All IR spectra were recorded on a Nicolet Impact-400 Fourier transform infrared spectrometer (FTIR) and the data are reported in reciprocal centimetres $\left(\mathrm{cm}^{-1}\right)$.

Melting points were determined with a Büchi B35 apparatus or a Kofler bank and were uncorrected.

Low resolution mass spectra (LRMS) were recorded on a Brüker Esquire 3000 plus (ESI). High resolution mass spectra (HRMS) were recorded at the "Service Central d'Analyses du CNRS", Vernaison, France or at the “Centre Régional de Mesures Physiques de l’Ouest”, Rennes, France.

Elemental analyses were performed at the "Service Central d'Analyses du CNRS", Vernaison, France.

Reactions were performed using oven dried glasswares under an atmosphere of dry argon. They were monitored by thin layer chromatography (TLC) using commercial aluminium-backed silica gel plates (Merck, Kieselgel 60 $\mathrm{PF}_{254}$ ). TLC spots were viewed under ultraviolet light and by heating the plate after treatment with an appropriate revelatory (ninhydrin, $\mathrm{KMnO}_{4}$, TTC).

Chromatography purifications were performed by gravity column chromatography using Macherey-Nagel Silica Gel 60 (70-230 mesh).

Solvents: Tetrahydrofuran and diethyl ether were refluxed on sodium-benzophenone and then distilled. Dichloromethane and methanol were dried by refluxing on $\mathrm{CaH}_{2}$ and then distilled. Unless otherwise noted, all reagent-grade chemicals and solvents were obtained from commercial suppliers (Sigma-Aldrich, Acros Organics and Avocado) and were used as received. 


\section{Synthesis of tert-butyl (phenylsulfonyl)alkyl- $N$-hydroxycarbamates 1}

- tert-Butyl 3-methyl-1-(phenylsulfonyl)butyl- $N$-hydroxycarbamate $1 a^{1}$

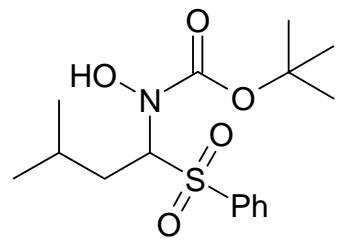

Sodium benzenesulfinate $(3.08 \mathrm{~g}, 18.8 \mathrm{mmol})$ was dissolved into $22.5 \mathrm{~mL}$ of a mixture of water/methanol (2/1). To this solution was added sequentially tert-butyl- $N$ hydroxycarbamate $(1 \mathrm{~g}, 7.52 \mathrm{mmol})$, of isovaleraldehyde $(1.293 \mathrm{~g}, 1.61 \mathrm{~mL}, 15.04 \mathrm{mmol})$ and formic acid $(0.57 \mathrm{~mL}, 15.04 \mathrm{mmol})$. This solution was stirred for 20 hours at room temperature. The resulting white precipitate was collected by filtration $(2.55 \mathrm{~g}, 7.42 \mathrm{mmol})$ and recrystallized from EtOAc. When the reaction was performed with only 1 equiv of aldehyde and 1.2 equiv of sodium benzenesulfinate, the yield decreased to $69 \%$.

Yield: 99\%. Mp: $102{ }^{\circ} \mathrm{C}$. IR (KBr): 3347, 2959, 2934, 2869, 1667, 1434, 1395, 1369, 1318, 1260, 1150, 1085, 852, $755 \mathrm{~cm}^{-1} .{ }^{\mathbf{1}} \mathbf{H} \mathbf{R M N}\left(\mathbf{C D C l}_{3}, \mathbf{3 0 0} \mathbf{M H z}\right): \delta=0.88(\mathrm{~d}, J=6.4 \mathrm{~Hz}, 3 \mathrm{H}$, $\left.\mathrm{CH}_{3}\right), 0.98\left(\mathrm{~d}, \mathrm{~J}=6.4 \mathrm{~Hz}, 3 \mathrm{H}, \mathrm{CH}_{3}\right), 1.28\left(\mathrm{~s}, 9 \mathrm{H}, \mathrm{C}\left(\underline{\mathrm{CH}}_{3}\right)_{3}\right), 1.65-1.85\left(\mathrm{~m}, 2 \mathrm{H}, \mathrm{CH}_{2}\right), 2.12-2.32$ (m, 1H, $\underline{\mathrm{CHMe}} 2$ ), 5.05-5.30 (m, 1H, CHN), 6.95 (br s, 1H, OH), 7.50-7.60 (m, 2H, H arom.), 7.60-7.68 (m, 1H, H arom.), 7.90-8.00 (m, 2H, H arom.). ${ }^{13} \mathbf{C} \mathbf{R M N}\left(\mathbf{C D C l}_{3}, \mathbf{7 5 . 5} \mathbf{~ M H z}\right): \delta=$ $\left.20.7\left(\mathrm{CH}_{3}\right), 23.1\left(\mathrm{CH}_{3}\right), 24.7(\mathrm{CH}), 27.8\left(\mathrm{C}^{\left(\mathrm{CH}_{3}\right.}\right)_{3}\right), 31.7\left(\mathrm{CH}_{2}\right), 83.3\left(\underline{\mathrm{C}}\left(\mathrm{CH}_{3}\right)_{3}, 124.9(\mathrm{CHN})\right.$, 129.0 ( $\mathrm{CH}$ arom.), 129.3 ( $\mathrm{CH}$ arom.), 133.9 ( $\mathrm{CH}$ arom.), 137.6 (C arom.), 154.5 (C=O). LRMS (ESI): $\mathrm{m} / \mathrm{z}=344\left[(\mathrm{M}+\mathrm{H})^{+}\right], 318,272$. HRMS (ESI) calcd for $\mathbf{C}_{\mathbf{1 6}} \mathbf{H}_{\mathbf{2 5}} \mathbf{N O}_{5} \mathbf{N a S}$ $\left[(\mathrm{M}+\mathrm{Na})^{+}\right]:$366.1351. Found: 366.1356 . Anal. Calcd for $\mathbf{C}_{\mathbf{1 6}} \mathbf{H}_{25} \mathbf{N O}_{5} \mathbf{S}: \mathbf{C}, 55.96 ; \mathrm{H}, 7.34 ; \mathrm{N}$, 4.08. Found: C, 55.95; H, 7.42; N, 3.91.

\section{tert-Butyl 2- $N^{\prime}$-[(tert-butoxycarbonyl)amino]-1-(phenylsulfonyl)ethyl- $N$ - hydroxycarbamate $1 b$}

\footnotetext{
${ }^{1}$ Guinchard, X.; Vallée, Y.; Denis, J.-N. Org. Lett. 2005, 23, 5147.
} 


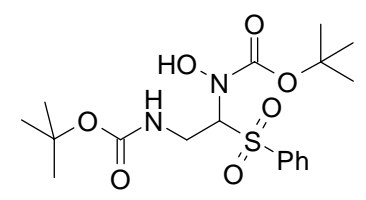

Sodium benzenesulfinate $(8.59 \mathrm{~g}, 52.36 \mathrm{mmol})$ was dissolved into $76 \mathrm{~mL}$ of a mixture of water/THF (2/1). To this solution was added sequentially tert-butyl- $N$-hydroxycarbamate (3.48 g, $26.18 \mathrm{mmol}), N$-(tert-butoxycarbonyl)aminoacetaldehyde (4.11 g, $26.18 \mathrm{mmol}$ ) and formic acid (1.04 g, $26.18 \mathrm{mmol})$. This solution was stirred for 20 hours at room temperature. It was then extracted twice with ethyl acetate. Combined organic layers were washed with brine, dried over anhydrous magnesium sulfate and evaporated. The sulfone $\mathbf{1 b}$ was obtained as a white solid $(6.50 \mathrm{~g}, 15.63 \mathrm{mmol})$.

Yield: 60\%. Mp: 123.5-124.5 ${ }^{\circ} \mathrm{C}$. IR (KBr): 3360, 2988, 2932, 1712, 1672, 1526, 1373, 1324, 1155, $1114 \mathrm{~cm}^{-1} .{ }^{1} \mathbf{H} \mathbf{R M N}\left(\mathbf{C D C l}_{3}, 300 \mathbf{M H z}\right): \delta=1.25-1.55\left(\mathrm{~m}, 18 \mathrm{H}, 2 \mathrm{C}\left(\underline{\mathrm{CH}}_{3}\right)_{3}\right)$, 3.65-4.05 (m, $\left.2 \mathrm{H}, \mathrm{CH}_{2} \mathrm{~N}\right)$, 5.05-5.20 (m, 1H, CHN), 5.20-5.45 (m, 1H, NHBoc), 7.50-7.60 (m, 2H, H arom.), 7.60-7.70 (m, 1H, H arom.), 7.95-8.05 (m, 2H, H arom.). ${ }^{13} \mathbf{C} \mathbf{R M N}$ $\left(\mathbf{C D C l}_{3}, 75.5 \mathrm{MHz}\right): \delta=27.9\left(\mathrm{C}\left(\underline{\mathrm{CH}}_{3}\right)_{3}\right), 28.1\left(\mathrm{C}\left(\underline{\mathrm{CH}}_{3}\right)_{3}\right), 35.3\left(\mathrm{CH}_{2} \mathrm{~N}\right), 81.2\left(\underline{\mathrm{C}}\left(\mathrm{CH}_{3}\right)_{3}\right), 82.4$ $\left(\underline{\mathrm{C}}\left(\mathrm{CH}_{3}\right)_{3}\right), 124.8(\mathrm{CHN}), 129.0$ (CH arom.), 129.4 (CH arom.), 134.1 ( $\mathrm{CH}$ arom.), 137.2 (C arom.), 157.5 (2 $\mathrm{C}=\mathrm{O})$. LRMS (ESI, 1\% LiCl): m/z= $423\left[(\mathrm{M}+\mathrm{Li})^{+}\right], 839\left[(\operatorname{dimer}+\mathrm{Li})^{+}\right]$. HRMS (ESI) calcd for $\mathbf{C}_{8} \mathbf{H}_{14} \mathbf{N}_{2} \mathbf{O}_{5}:$ 218.0903. Found: 218.0879 [(M-PhSO ${ }_{2} \mathrm{H}-t-$ $\left.\mathrm{Bu}+\mathrm{H})^{+}\right]$.Anal. Calcd for $\mathbf{C}_{\mathbf{1 8}} \mathbf{H}_{\mathbf{2}} \mathbf{N}_{\mathbf{2}} \mathbf{O}_{7} \mathrm{~S}: \mathrm{C}, 51.91 ; \mathrm{H}, 6.78 ; \mathrm{N}, 6.73$. Found: C, 51.91; $\mathrm{H}$, $6.86 ; \mathrm{N}, 6.66$.

\section{- tert-Butyl 2-(benzyloxy)-1-(phenylsulfonyl)ethyl- $N$-hydroxycarbamate 1c}

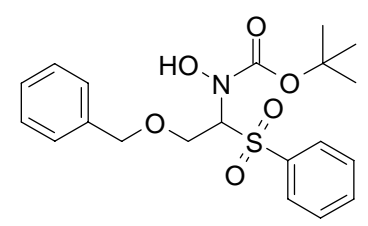

Sodium benzenesulfinate $(1.53 \mathrm{~g}, 9.32 \mathrm{mmol})$ was dissolved into $11 \mathrm{~mL}$ of a mixture of $\mathrm{H}_{2} \mathrm{O} / \mathrm{MeOH}(2 / 1)$. To this solution was added sequentially tert-butyl- $N$-hydroxycarbamate (500 $\mathrm{mg}, 3.76 \mathrm{mmol}$ ), benzyloxyacetaldehyde $(1.06 \mathrm{~mL}, 7.52 \mathrm{mmol})$ and then formic acid (300 mg, $7.52 \mathrm{mmol}$ ). This solution was then stirred for 20 hours at room temperature. The resulting white precipitate was collected by filtration and dried under reduced pression. The 
sulfone 1c was recrystallized from $\mathrm{MeOH} / \mathrm{H}_{2} \mathrm{O}$ and obtained as a white solid (730 $\mathrm{mg}, 1.79$ mmol).

Yield: 65\%. IR (neat): 3400, 2975, 2910, 1712, 1453, 1368, 1305, 1151, 1121, $1085 \mathrm{~cm}^{-1} .{ }^{\mathbf{1}} \mathbf{H}$ NMR (CDCl 3300 MHz): $\delta=1.38\left(\mathrm{~s}, 9 \mathrm{H}, \mathrm{C}\left(\underline{\mathrm{CH}}_{3}\right)_{3}\right), 4.14\left(\mathrm{~d}, \mathrm{~J}=6.7 \mathrm{~Hz}, 2 \mathrm{H}, \mathrm{CH}_{2}\right), 4.53$ (s, $2 \mathrm{H}, \mathrm{CH}_{2}$ ), 5.41 (bs, 1H, CHN), 7.20-7.38 (m, 5H, H arom), 7.48-7.58 (m, 2H, H arom), 7.617.69 (m, 1H, H arom), 7.88-7.94 (m, 2H, H arom). ${ }^{13} \mathbf{C}$ NMR (CDCl $\left.\mathbf{3}, \mathbf{7 5 . 5} \mathbf{~ M H z}\right): \delta=27.9$ $\left(\mathrm{C}\left(\underline{\mathrm{CH}}_{3}\right)_{3}\right), 63.6\left(\mathrm{CH}_{2}\right), 73.5\left(\mathrm{CH}_{2}\right), 83.7\left(\underline{\mathrm{C}}\left(\mathrm{CH}_{3}\right)_{3}\right), 127.7,127.9$ and $128.4(\mathrm{CHN}$ and $\mathrm{CH}$ arom), 129.1 (CH arom), 134.0 ( $\mathrm{CH}$ arom), 137.0 (C arom), $138.1(\mathrm{C}$ arom), $155.1(\mathrm{C}=\mathrm{O})$. LRMS (ESI): $\mathrm{m} / \mathrm{z}=288\left[\left(\mathrm{M}-\mathrm{PhSO}_{2} \mathrm{H}\right)^{+}\right]$. Anal. Calcd for $\mathbf{C}_{20} \mathbf{H}_{25} \mathbf{N O}_{6} \mathbf{S}: \mathrm{C}, 58.96 ; \mathrm{H}, 6.19$; N, 3.44. Found: C, 58.91; H, 6.13; N, 3.59.

\section{- tert-Butyl 1-cyclohexyl-1-(phenylsulfonyl)methyl- $N$-hydroxycarbamate 1d}

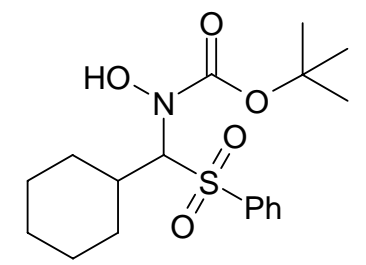

Sodium benzenesulfinate $(3.08 \mathrm{~g}, 18.8 \mathrm{mmol})$ was dissolved into $22.5 \mathrm{~mL}$ of a mixture of water/THF (2/1). To this solution was added sequentially tert-butyl- $N$-hydroxycarbamate (1 g, $7.52 \mathrm{mmol})$, of cyclohexanecarboxaldehyde $1.69 \mathrm{~g}(15.04 \mathrm{mmol})$ and formic acid $(0.57$ $\mathrm{mL}, 15.04 \mathrm{mmol}$ ). This solution was stirred for 20 hours at room temperature. The resulting white precipitate was collected by filtration and washed with water and pentane. The obtained white solid (2.45 g, $6.64 \mathrm{mmol})$ was recrystallized from EtOAc.

Yield: 89 \%. Mp: $124{ }^{\circ} \mathrm{C}$. IR (KBr): 3320, 3069, 2980, 2924, 2851, 1664, 1454, 1389, 1308, 1147, 1082, $847 \mathrm{~cm}^{-1} .{ }^{\mathbf{1}} \mathbf{H}$ NMR (CD $\left.\mathbf{C O C D}_{3}, \mathbf{3 0 0} \mathbf{M H z}\right): \delta=1.29\left(\mathrm{~s}, 9 \mathrm{H}, \mathrm{C}\left(\underline{\mathrm{CH}}_{3}\right)_{3}\right), 1.40-$ $1.50\left(\mathrm{~m}, 3 \mathrm{H}, \mathrm{CH}\right.$ and $\left.\mathrm{CH}_{2}\right), 1.55-1.65\left(\mathrm{~m}, 4 \mathrm{H}, \mathrm{CH}_{2}\right), 1.70-1.83\left(\mathrm{~m}, 2 \mathrm{H}, \mathrm{CH}_{2}\right), 2.20-2.40(\mathrm{~m}$, 2H, $\mathrm{CH}_{2}$ ), 4.95-5.25 (m, 1H, CHN), 7.50-7.65 (m, 2H, H arom.), 7.65-7.78 (m, 1H, H arom.), 7.95-8.05 (m, 2H, H arom.). ${ }^{13} \mathbf{C}$ NMR ( $\left.\mathbf{C D C l}_{3}, 75.5 \mathbf{M H z}\right): \delta=25.4\left(\mathrm{CH}_{2}\right), 25.6\left(\mathrm{CH}_{2}\right), 25.9$ $\left(\mathrm{CH}_{2}\right), 27.9\left(\mathrm{C}\left(\underline{\mathrm{CH}}_{3}\right)_{3}\right), 29.7\left(\mathrm{CH}_{2}\right), 30.2\left(\mathrm{CH}_{2}\right), 35.6(\mathrm{CH}), 83.6\left(\underline{\mathrm{C}}\left(\mathrm{CH}_{3}\right)_{3}\right), 124.9(\mathrm{CHN})$, 129.0 (CH arom.), 131.8 (CH arom.), 133.7 (CH arom.), 154.8 (C=O). LRMS (ESI, MeOH): 
$\mathrm{m} / \mathrm{z}=455\left[\left(\mathrm{MH}-\mathrm{PhSO}_{2} \mathrm{H} \text { dimer }\right)^{+}\right], 228\left[(\text { nitrone }+\mathrm{H})^{+}\right]$. Anal. Calcd for $\mathbf{C}_{\mathbf{1 8}} \mathbf{H}_{\mathbf{2}} \mathbf{N O}_{\mathbf{5}} \mathbf{S}: \mathbf{C}$, 58.51; H, 7.37; N, 3.79. Found: C, 58.53; H, 7.51; N, 3.80.

- tert-Butyl 1-cyclohex-2-enyl-1-(phenylsulfonyl)methyl- $N$-hydroxycarbamate $1 \mathrm{e}$

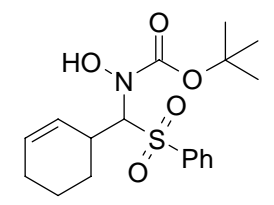

Sodium benzenesulfinate $(3.08 \mathrm{~g}, 18.8 \mathrm{mmol})$ was dissolved into $22 \mathrm{~mL}$ of a mixture of water/methanol (2/1). To this solution was added sequentially tert-butyl- $N$ hydroxycarbamate (1 g, $7.52 \mathrm{mmol}), 3$-cyclohexene-1-carboxaldehyde (1.654 g, $15.04 \mathrm{mmol})$ and formic acid $(690 \mathrm{mg}, 15.04 \mathrm{mmol})$. This solution was stirred for 7 hours at room temperature. It was then filtered, washed with water and pentane and dried. The product 1e was obtained as a white solid (1.865 g, $5.08 \mathrm{mmol})$.

Yield: 68\%. IR (film): 3324, 3068, 2984, 2954, 2921, 2838, 1662, 1443, 1399, 1373, 1319, 1374, 1253, 1153, 1120, 1083, $847 \mathrm{~cm}^{-1} .{ }^{\mathbf{1}} \mathbf{H}$ NMR (CDCl 3 , $\left.300 \mathbf{M H z}\right): \delta=1.10-1.60$ (m, 9H, $\left.\mathrm{C}\left(\underline{\mathrm{CH}}_{3}\right)_{3}\right), 1.80-2.20\left(\mathrm{~m}, 4 \mathrm{H}, \mathrm{CH}_{2}\right), 2.20-2.35\left(\mathrm{~m}, 2 \mathrm{H}, \mathrm{CH}_{2}\right), 2.40-2.95(\mathrm{~m}, 1 \mathrm{H}, \mathrm{CH}), 4.90-5.10$ and 5.20-5.40 (m, 1H, CHN), 5.50-5.75 (m, 2H, CH), $7.13(\mathrm{bs}, 1 \mathrm{H}, \mathrm{OH}), 7.50-7.58(\mathrm{~m}, 2 \mathrm{H}, \mathrm{H}$ arom), 7.60-7.68 (m, 1H, H arom), 7.90-8.02 (m, 2H, H arom). ${ }^{13} \mathbf{C}$ NMR $\left(\mathbf{C D C l}_{3}, \mathbf{7 5 . 5}\right.$ MHz): $\delta=23.7$ and $23.8\left(\mathrm{CH}_{2}\right), 25.5$ and $25.6\left(\mathrm{CH}_{2}\right), 27.9\left(\mathrm{C}\left(\underline{\mathrm{CH}}_{3}\right)_{3}\right), 28.2$ and $28.4\left(\mathrm{CH}_{2}\right)$, 31.3 and $31.7(\mathrm{CH}), 83.7\left(\underline{\mathrm{C}}\left(\mathrm{CH}_{3}\right)_{3}\right), 124.7(\mathrm{CH}), 124.9(\mathrm{CH}), 126.8(\mathrm{CH}), 129.0(\mathrm{CH}$ arom $)$, 131.9 (CH arom), 133.9 (CH arom), 154.9 and $155.0(\mathrm{C}=\mathrm{O})$. LRMS (ESI): $\mathrm{m} / \mathrm{z}=248$ [(M$\left.\left.\mathrm{PhSO}_{2} \mathrm{H}+\mathrm{Na}\right)^{+}\right], \quad 264 \quad\left[\left(\mathrm{M}-\mathrm{PhSO}_{2} \mathrm{H}+\mathrm{K}\right)^{+}\right], \quad 390 \quad\left[(\mathrm{M}+\mathrm{Na})^{+}\right], \quad 406 \quad\left[(\mathrm{M}+\mathrm{K})^{+}\right], 473 \quad[2(\mathrm{M}-$ $\left.\left.\left.\mathrm{PhSO}_{2} \mathrm{H}\right)+\mathrm{Na}\right)^{+}\right], 489\left[2\left(\mathrm{M}-\mathrm{PhSO}_{2} \mathrm{H}+\mathrm{K}\right)^{+}\right]$. Anal. Calcd for $\mathbf{C}_{\mathbf{1 8}} \mathbf{H}_{\mathbf{2}} \mathbf{N O} \mathbf{O}_{\mathbf{5}} \mathrm{S}$ : C, 58.84; $\mathrm{H}, 6.86$; N, 3.82. Found: C, 58.71; H, 6.89; N, 3.59.

\section{- tert-Butyl 1-(phenylsulfonyl)ethyl- $N$-hydroxycarbamate 1f}

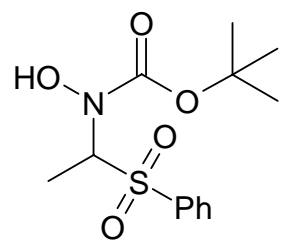


Sodium benzenesulfinate $(3.08 \mathrm{~g}, 18.8 \mathrm{mmol})$ was dissolved into $22 \mathrm{~mL}$ of a mixture of water/methanol (2/1). To this solution was added sequentially tert-butyl- $N$ hydroxycarbamate $(1 \mathrm{~g}, 7.52 \mathrm{mmol})$, acetaldehyde $(660 \mathrm{mg}, 15.04 \mathrm{mmol})$ and formic acid (690 mg, $15.04 \mathrm{mmol}$ ). This solution was stirred for 7 hours at room temperature. It was then filtered, washed with water and pentane and dried. Product 1f was obtained as a white solid $(1.69 \mathrm{~g}, 5.64 \mathrm{mmol})$ and recrystallized from EtOAc.

Yield: 75\%. Mp: $124{ }^{\circ} \mathrm{C}$. IR (KBr): 3334, 2983, 2935, 1711, 1474, 1445, 1364, 1293, 1250 , 1146, 1127, 1046, $866 \mathrm{~cm}^{-1} .{ }^{1} \mathbf{H}$ NMR (CDCl $\left.3,300 \mathbf{~ M H z}\right): \delta=1.33\left(\mathrm{~s}, 9 \mathrm{H}, \mathrm{C}\left(\underline{\mathrm{CH}}_{3}\right)_{3}\right), 1.68(\mathrm{~d}$, $\left.J=7.1 \mathrm{~Hz}, 3 \mathrm{H}, \mathrm{CH}_{3}\right), 5.31(\mathrm{q}, J=6.8 \mathrm{~Hz}, 1 \mathrm{H}, \mathrm{CHN}), 6.92(\mathrm{br} \mathrm{s}, 1 \mathrm{H}, \mathrm{OH}), 7.50-7.60(\mathrm{~m}, 2 \mathrm{H}, \mathrm{H}$ arom.), 7.62-7.70 (m, 1H, CH arom.), 7.90-8.00 (m, 2H, H arom.). ${ }^{13} \mathbf{C}$ NMR (CDCl $\mathbf{3}, \mathbf{7 5 . 5}$ MHz): $\delta=10.6\left(\mathrm{CH}_{3}\right), 27.9\left(\mathrm{C}\left(\underline{\mathrm{CH}}_{3}\right)_{3}\right), 83.4\left(\left(\underline{\mathrm{C}}\left(\mathrm{CH}_{3}\right)_{3}\right), 124.9(\mathrm{CHN}), 129.0(\mathrm{CH}\right.$ arom. $)$, 129.1 ( $\mathrm{CH}$ arom.), 129.4 ( $\mathrm{CH}$ arom.), 134.0 ( $\mathrm{CH}$ arom.), 137.2 (C arom.), 154.6 (C=O). LRMS (ESI): $\mathrm{m} / \mathrm{z}=324\left[(\mathrm{M}+\mathrm{Na})^{+}\right], 341\left[(\text { nitrone dimer }+\mathrm{Na})^{+}\right]$. Anal. Calcd for $\mathrm{C}_{13} \mathrm{H}_{19} \mathrm{NO}_{5} \mathrm{~S}: \mathrm{C}, 51.82 ; \mathrm{H}, 6.36 ; \mathrm{N}, 4.65$. Found: $\mathrm{C}, 51.56 ; \mathrm{H}, 6.55 ; \mathrm{N}, 4.62$. 


\section{Addition of thienylmagnesium bromide: preparation of $\alpha$-thienyl- $N$-hydroxylamines 4}

\section{General procedure}

To a stirred solution of sulfone $\mathbf{1}$ in toluene under inert atmosphere were added two or three equiv of 2-thienylmagnesium bromide at room temperature. The resulting mixture was stirred during $45 \mathrm{~min}$ before being quenched by addition of an aqueous saturated solution of ammonium chloride. The mixture was then extracted three times with EtOAc. Organic layers were washed with brine and dried over anhydrous magnesium sulfate. After the removal of the solvent, the crude mixture was then purified by column chromatography on silica gel (Eluent: $\mathrm{CH}_{2} \mathrm{Cl}_{2}$ ) to give the pure product 4 .

- 1-[N-(tert-butoxycarbonyl)- $N$-(hydroxy)amino]-2-[ $N$-(tert-butoxycarbonyl)]-1(thien-2-yl)ethane $4 b$

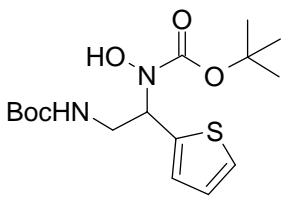

Prepared according to the above procedure, by reaction of sulfone 1b (200 mg, 0.48 mmol) with three equiv of thienylmagnesium bromide $(1.44 \mathrm{~mL}, 1.44 \mathrm{mmol}, 1 \mathrm{M}$ solution in toluene) in $4 \mathrm{~mL}$ of toluene. The product $\mathbf{4 b}$ was obtained as an oil (127 mg, $0.35 \mathrm{mmol})$.

Yield: 74\%. IR (film): 3304, 3004, 2974, 2944, 1709, 1699, 1666, 1523, 1393, 1366, 1289, 1246, 1173, $1113 \mathrm{~cm}^{-1} .{ }^{1} \mathbf{H}$ NMR (CDCl 3 , 300 MHz): $\delta=1.45$ (s, 18H, $\left.2 \mathrm{C}\left(\underline{\mathrm{CH}}_{3}\right)_{3}\right), 3.25-3.38$ $\left(\mathrm{m}, 1 \mathrm{H}, \mathrm{H}\right.$ of $\left.\mathrm{CH}_{2} \mathrm{~N}\right), 3.76-3.95\left(\mathrm{~m}, 1 \mathrm{H}, \mathrm{H}\right.$ of $\left.\mathrm{CH}_{2} \mathrm{~N}\right), 5.25-5.34(\mathrm{~m}, 1 \mathrm{H}, \mathrm{NHBoc}), 5.38$ (dd, $J=$ 4.2 and $11.3,1 \mathrm{H}, \mathrm{CHN}), 6.95(\mathrm{dd}, J=3.6$ and $5.0 \mathrm{~Hz}, 1 \mathrm{H}, \mathrm{H}$ arom), 7.04 (d, $J=3.6 \mathrm{~Hz}, 1 \mathrm{H}, \mathrm{H}$ arom), $7.22(\mathrm{dd}, J=1.2$ and $5.0 \mathrm{~Hz}, 1 \mathrm{H}, \mathrm{H}$ arom), 8.02 (br s, $1 \mathrm{H}, \mathrm{OH}) .{ }^{{ }^{13} \mathbf{C}} \mathbf{~ N M R}\left(\mathbf{C D C l}_{3}\right.$, $75.5 \mathrm{MHz}): \delta=28.3\left(2 \mathrm{C}\left(\underline{\mathrm{CH}}_{3}\right)_{3}\right), 42.1\left(\mathrm{CH}_{2} \mathrm{~N}\right), 58.0(\mathrm{CHN}), 80.5\left(\underline{\mathrm{C}}\left(\mathrm{CH}_{3}\right)_{3}\right), 81.6\left(\underline{\mathrm{C}}\left(\mathrm{CH}_{3}\right)_{3}\right)$, $125.0(\mathrm{CH}$ arom), $125.6(\mathrm{CH}$ arom $), 126.4(\mathrm{CH}$ arom $), 138.9(\mathrm{C}$ arom $), 156.5(\mathrm{C}=\mathrm{O}), 157.7$ $(\mathrm{C}=\mathrm{O})$. LRMS (ESI): $\mathrm{m} / \mathrm{z}=381\left[(\mathrm{M}+\mathrm{Na})^{+}\right], 397\left[(\mathrm{M}+\mathrm{K})^{+}\right]$.

- 1-[N-(tert-butoxycarbonyl)- $N$-(hydroxy)amino]-2-(benzyloxy)-1-(thien-2yl)ethane 4c

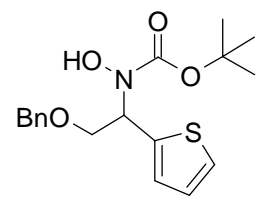


Prepared according to the above procedure, by reaction of sulfone $1 \mathrm{c}$ ( $200 \mathrm{mg}, 0.49$ mmol) with two equiv of thienylmagnesium bromide $(0.99 \mathrm{~mL}, 0.99 \mathrm{mmol}, 1 \mathrm{M}$ solution in toluene) in $4 \mathrm{~mL}$ of toluene. The product $4 \mathrm{c}$ was obtained as an oil ( $80 \mathrm{mg}, 0.23 \mathrm{mmol})$.

Yield: 47\%. IR (film): 3281, 2978, 2933, 2868, 1699, 1459, 1394, 1369, 1327, 1171, 1108

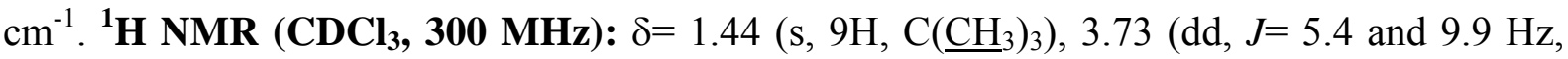
$1 \mathrm{H}, \mathrm{H}$ of $\left.\mathrm{CH}_{2}\right), 4.08\left(\mathrm{t}, J=9.9 \mathrm{~Hz}, 1 \mathrm{H}, \mathrm{H}\right.$ of $\left.\mathrm{CH}_{2}\right), 4.59\left(\mathrm{~s}, 2 \mathrm{H}, \mathrm{CH}_{2}\right), 5.56(\mathrm{dd}, J=5.2$ and 9.2 $\mathrm{Hz}, 1 \mathrm{H}, \mathrm{CHN}), 6.94$ (dd, $J=3.6$ and $5.1 \mathrm{~Hz}, 1 \mathrm{H}, \mathrm{H}$ arom), 7.00-7.05 (m, 1H, H arom), 7.21 (dd, $J=1.2$ and $5,1 \mathrm{~Hz}, 1 \mathrm{H}, \mathrm{H}$ arom), 7.25-7.40 (m, 5H, $\mathrm{H}$ arom). ${ }^{13} \mathbf{C}$ NMR $\left(\mathbf{C D C l}_{3}, \mathbf{7 5 . 5}\right.$ MHz): $\delta=28.2\left(\mathrm{C}\left(\underline{\mathrm{CH}}_{3}\right)_{3}\right), 58.1(\mathrm{CHN}), 69.4\left(\mathrm{CH}_{2}\right), 73.2\left(\mathrm{CH}_{2}\right), 82.5\left(\underline{\mathrm{C}}\left(\mathrm{CH}_{3}\right)_{3}\right), 125.0(\mathrm{CH}$ arom), $125.8(\mathrm{CH}$ arom), $126.5(\mathrm{CH}$ arom $), 127.7(\mathrm{CH}$ arom $), 127.8(\mathrm{CH}$ arom $), 128.4(\mathrm{CH}$ arom), 137.7 (C arom), 138.7 (C arom), 157.3 (C=O). LRMS (ESI): $\mathrm{m} / \mathrm{z}=372\left[(\mathrm{M}+\mathrm{Na})^{+}\right]$, $388\left[(\mathrm{M}+\mathrm{K})^{+}\right]$. HRMS (ESI) calcd for $\mathbf{C}_{\mathbf{1 8}} \mathbf{H}_{\mathbf{2}} \mathbf{N O}_{4} \mathbf{S N a}\left[(\mathrm{M}+\mathrm{Na})^{+}\right]$: 372.1246 . Found: 372.1251 .

- 1-[N-(tert-butoxycarbonyl)- $N$-(hydroxy)amino]-2-(cyclohexyl)-1-(thien-2yl)methane 4d

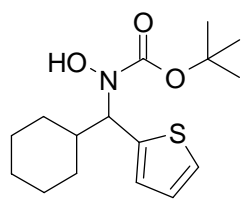

Prepared according to the above general procedure, by reaction of sulfone $1 \mathbf{1}$ (300 $\mathrm{mg}$, $0.82 \mathrm{mmol})$ with two equiv of thienylmagnesium bromide $(1.63 \mathrm{~mL}, 1.63 \mathrm{mmol}, 1 \mathrm{M}$ solution in toluene) in $5 \mathrm{~mL}$ of toluene. The product $\mathbf{4 d}$ was obtained as an oil (135 $\mathrm{mg}, 0.44 \mathrm{mmol})$.

Yield: 54\%. IR (film): 3202, 2966, 2944, 2842, 1685, 1400, 1366, 1329, 1168, $1109 \mathrm{~cm}^{-1}$. ${ }^{\mathbf{1}} \mathbf{H}$ NMR (CDCl 3,300 MHz): $\delta=0.75-0.90\left(\mathrm{~m}, 1 \mathrm{H}, \mathrm{CH}\right.$ of $\left.\mathrm{CH}_{2}\right), 0.90-1.10\left(\mathrm{~m}, 1 \mathrm{H}, \mathrm{CH}\right.$ of $\left.\mathrm{CH}_{2}\right)$, 1.10-1.35 (m, 3H, $\mathrm{CH}$ and $\left.\mathrm{CH}_{2}\right), 1.41\left(\mathrm{~s}, 9 \mathrm{H}, \mathrm{C}\left(\underline{\mathrm{CH}}_{3}\right)_{3}\right), 1.55-1.68\left(\mathrm{~m}, 2 \mathrm{H}, \mathrm{CH}_{2}\right), 1.68-1.80$ $\left(\mathrm{m}, 1 \mathrm{H}, \mathrm{CH}\right.$ of $\left.\mathrm{CH}_{2}\right), 1.95-2.15\left(\mathrm{~m}, 2 \mathrm{H}, \mathrm{CH}_{2}\right), 4.82(\mathrm{~d}, J=10.6 \mathrm{~Hz}, 1 \mathrm{H}, \mathrm{CHN}), 6.92(\mathrm{dd}, J=$ 3.5 and $5.0 \mathrm{~Hz}, 1 \mathrm{H}, \mathrm{H}$ arom), 6.98 (dd, $J=1.2$ and $3.5 \mathrm{~Hz}, 1 \mathrm{H}, \mathrm{H}$ arom), 7.17 (dd, $J=1.0$ and $5.1 \mathrm{~Hz}, 1 \mathrm{H}, \mathrm{H}$ arom), 7.40 (br s, $1 \mathrm{H}, \mathrm{OH}) .{ }^{13} \mathbf{C} \mathbf{N M R}\left(\mathbf{C D C l}_{3}, \mathbf{7 5 . 5} \mathbf{~ M H z}\right): \delta=25.7\left(\mathrm{CH}_{2}\right)$, $25.8\left(\mathrm{CH}_{2}\right), 26.3\left(\mathrm{CH}_{2}\right), 28.2\left(\mathrm{C}\left(\underline{\mathrm{CH}}_{3}\right)_{3}\right), 30.1\left(\mathrm{CH}_{2}\right), 30.4\left(\mathrm{CH}_{2}\right), 40.1(\mathrm{CH}), 63.6(\mathrm{CHN}), 81.9$ $\left(\underline{\mathrm{C}}\left(\mathrm{CH}_{3}\right)_{3}\right), 124.5(\mathrm{CH}$ arom, $125.8(\mathrm{CH}$ arom $), 126.0(\mathrm{CH}$ arom $), 140.7(\mathrm{C}$ arom $), 157.0$ $(\mathrm{C}=\mathrm{O})$. LRMS (ESI): $\mathrm{m} / \mathrm{z}=334\left[(\mathrm{M}+\mathrm{Na})^{+}\right]$. Anal. Calcd for $\mathbf{C}_{\mathbf{1 6}} \mathbf{H}_{\mathbf{2 5}} \mathbf{N O}_{3} \mathrm{~S}: \mathrm{C}, 61.71 ; \mathrm{H}, 8.09$; N, 4.50. Found: C, 61.48; H, 8.22; N, 4.43.

- 1-[N-(tert-butoxycarbonyl)- $N$-(hydroxy)amino]-2-[cyclohex-2-enyl]-1-(thien-2yl)methane 4e 


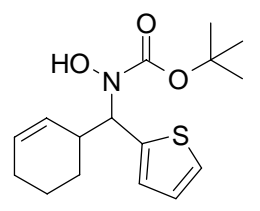

Prepared according to the above general procedure, by reaction of sulfone 1e $(200 \mathrm{mg}$, $0.55 \mathrm{mmol})$ with two equiv of thienylmagnesium bromide $(1.09 \mathrm{~mL}, 1.09 \mathrm{mmol}, 1 \mathrm{M}$ solution in toluene) in $4 \mathrm{~mL}$ of toluene. The product $4 \mathbf{e}$ was obtained as an oil (108 $\mathrm{mg}, 0.35 \mathrm{mmol})$.

Yield: 64\%. IR (film): 3213, 3022, 2974, 2910, 2835, 1692, 1479, 1453, 1434, 1393, 1370, 1325, 1247, 1164, 1104, $853 \mathrm{~cm}^{-1} .{ }^{\mathbf{1}} \mathbf{H} \mathbf{~ N M R}\left(\mathbf{C D C l}_{3}, 300 \mathbf{M H z}\right): \delta=1.44\left(\mathrm{~s}, 9 \mathrm{H}, \mathrm{C}\left(\underline{\mathrm{CH}}_{3}\right)_{3}\right)$, 1.80-2.20 (m, 6H, $\left.\mathrm{CH}_{2}\right), 2.30-2.50(\mathrm{~m}, 2 \mathrm{H}), 4.93$ (t, J= $\left.9.5 \mathrm{~Hz}, \mathrm{CHN}\right), 5.50-5.75(\mathrm{~m}, 2 \mathrm{H}$, $\mathrm{CH}), 6.56$ (br s, 1H, OH), 6.92-7.04 (m, 2H, H arom), 7.20-7.25 (m, 1H, H arom). ${ }^{13} \mathrm{C}$ NMR $\left(\mathrm{CDCl}_{3}, 75.5 \mathrm{MHz}\right): \delta=24.3$ and $24.6\left(\mathrm{CH}_{2}\right), 25.4$ and $\left.25.8\left(\mathrm{CH}_{2}\right), 28.2\left(\mathrm{C}_{\left(\mathrm{CH}_{3}\right.}\right)_{3}\right), 29.0$ $\left(\mathrm{CH}_{2}\right), 35.9$ and $36.4(\mathrm{CH}), 62.3$ and $62.7(\mathrm{CHN}), 82.1\left(\underline{\mathrm{C}}\left(\mathrm{CH}_{3}\right)_{3}\right), 124.7(\mathrm{CH}), 125.5$ and $125.8(\mathrm{CH}), 125.9,125.97,126.00$ and $126.1(\mathrm{CH}), 126.7$ and $127.0(\mathrm{CH}), 140.3$ and $140.5(\mathrm{C}$ arom), 157.00 and $157.04(\mathrm{C}=\mathrm{O})$. LRMS (ESI): $\mathrm{m} / \mathrm{z}=308$ [(M-H)]. HRMS (ESI) calcd for $\mathrm{C}_{16} \mathrm{H}_{23} \mathrm{NO}_{3} \mathrm{NaS}\left[(\mathrm{M}+\mathrm{Na})^{+}\right]$: 332.1296 . Found: 332.1297 . 


\section{Preparation of furyl- $N$-hydroxylamine $4 f$}

\section{- 1-[N-(tert-butoxycarbonyl)- $N$-(hydroxy)amino]-3-methyl-1-(fur-2-yl)butane 4f}

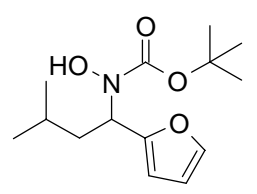

To a solution of furane ( $50 \mathrm{mg}, 0.73 \mathrm{mmol})$ and TMEDA ( $85 \mathrm{mg}, 0.73 \mathrm{mmol}$ ) in ether (1 $\mathrm{mL})$ at $0{ }^{\circ} \mathrm{C}$ was added $n$-BuLi $(1.28 \mathrm{M}$ in hexane, $0.57 \mathrm{~mL}, 0.73 \mathrm{mmol})$. After $30 \mathrm{~min}$, $\mathrm{MgBr}_{2}$. $\mathrm{OEt}_{2}(188 \mathrm{mg}, 0.73 \mathrm{mmol})$ was added and this solution was allowed to stir at room temperature. To this solution was added, $10 \mathrm{~min}$ later, the solution of the sulfone 1a $(100 \mathrm{mg}$, $0.29 \mathrm{mmol})$ in toluene $(2.5 \mathrm{~mL})$ and THF $(0.5 \mathrm{~mL})$. The resulting mixture was allowed to stir at room temperature during $1 \mathrm{~h}$. It was then quenched by addition of a saturated aqueous solution of ammonium chloride. Aqueous layer was extracted twice by EtOAc. Combined organic layers were washed by brine, dried over anhydrous magnesium sulfate and filtered. The solvents were removed and the residue was purified by column chromatography on flash silica gel (Eluent: $\mathrm{CH}_{2} \mathrm{Cl}_{2}$ ). The product $\mathbf{4 f}$ was obtained as colorless crystals (66 mg, 0.25 mmol).

Yield: 86\%. IR (film): 3213, 3150, 2962, 2869, 1681, 1408, 1389, 1325, 1157, 1101, 1011 $\mathrm{cm}^{-1} .{ }^{1} \mathbf{H}$ NMR (CDCl $\left.3,300 \mathrm{MHz}\right): \delta=0.90\left(\mathrm{~d}, J=4.6 \mathrm{~Hz}, 3 \mathrm{H}, \mathrm{CH}_{3}\right), 0.95(\mathrm{~d}, J=4.6 \mathrm{~Hz}, 3 \mathrm{H}$, $\left.\mathrm{CH}_{3}\right), 1.50$ (s, 9H, $\left.\mathrm{C}\left(\underline{\mathrm{CH}}_{3}\right)_{3}\right), 1.60-1.78\left(\mathrm{~m}, 2 \mathrm{H}, \mathrm{CH}_{2}\right), 1.95-2.10(\mathrm{~m}, 1 \mathrm{H}, \mathrm{CH}), 5.18$ (dd, J=5.4 and $10.2 \mathrm{~Hz}, 1 \mathrm{H}, \mathrm{CHN}), 6.24(\mathrm{~d}, J=3.3 \mathrm{~Hz}, 1 \mathrm{H}, \mathrm{H}$ arom), $6.31(\mathrm{dd}, J=1.8$ and $3.3 \mathrm{~Hz}, 1 \mathrm{H}, \mathrm{H}$ arom), 7.31 (d, $J=1.8 \mathrm{~Hz}, 1 \mathrm{H}, \mathrm{H}$ arom). ${ }^{13} \mathbf{C} \mathbf{~ N M R}\left(\mathbf{C D C l}_{3}, \mathbf{7 5 . 5} \mathbf{M H z}\right): \delta=21.7\left(\mathrm{CH}_{3}\right), 23.0$ $\left(\mathrm{CH}_{3}\right), 24.6(\mathrm{CH}), 28.2\left(\left(\underline{\mathrm{CH}}_{3}\right)_{3} \mathrm{C}\right), 38.5\left(\mathrm{CH}_{2}\right), 54.8(\mathrm{CHN}), 82.2\left(\underline{\mathrm{C}}\left(\mathrm{CH}_{3}\right)_{3}\right), 107.1(\mathrm{CH}$ arom), 110.1 (CH arom), $141.6(\mathrm{CH}$ arom), 153.5 (C arom), $157.1(\mathrm{C}=\mathrm{O})$. LRMS (ESI): $\mathrm{m} / \mathrm{z}=292\left[(\mathrm{M}+\mathrm{Na})^{+}\right], 561\left[(2 \mathrm{M}+\mathrm{Na})^{+}\right]$. Anal. Calcd for $\mathbf{C}_{\mathbf{1 4}} \mathbf{H}_{\mathbf{2} 3} \mathbf{N O}_{4}: \mathrm{C}, 62.44 ; \mathrm{H}, 8.61 ; \mathrm{N}$, 5.21. Found: C, 62.57; H, 8.90; N, 5.19. 


\section{Preparation of aromatic $N$-hydroxylamines 6}

\section{- $\quad$ 1-[ $N$-(tert-butoxycarbonyl)- $N$-(hydroxy)amino]-3-methyl-1-(4'- methoxyphenyl)butane 6a}

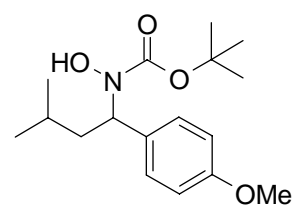

To a solution of sulfone 1a $(100 \mathrm{mg}, 0.29 \mathrm{mmol})$ in toluene $(3 \mathrm{~mL})$ and THF $(1 \mathrm{~mL})$ at room temperature under inert atmosphere was added 4-methoxyphenylmagnesium bromide 5a $(0.51 \mathrm{M}$ solution in toluene, $1.25 \mathrm{~mL}, 0.64 \mathrm{mmol})$. The reaction mixture was stirred at room temperature during 1 hour before being quenched by addition of a saturated aqueous solution of ammonium chloride. The resulting mixture was extracted twice by EtOAc. Combined organic layers were washed with brine, dried over anhydrous magnesium sulfate and the solvents were evaporated. The residue was purified by column chromatography on flash silica gel (Eluent: $\mathrm{CH}_{2} \mathrm{Cl}_{2}$ ). The product $6 \mathbf{a}$ was obtained as a white amorphous solid ( $87 \mathrm{mg}, 0.28$ mmol).

Yield: 97\%. IR (film): 3208, 2962, 2923, 2869, 1687, 1614, 1513, 1468, 1397, 1367, 1250, 1179, 1134, 1089, 1037, $835 \mathrm{~cm}^{-1} .{ }^{\mathbf{1}} \mathbf{H} \mathbf{N M R}\left(\mathbf{C D C l}_{3}, \mathbf{3 0 0} \mathbf{M H z}\right): \delta=0.95$ (d, J=6.4 Hz, 6H, 2 $\left.\mathrm{CH}_{3}\right), 1.44\left(\mathrm{~s}, 9 \mathrm{H},\left(\underline{\mathrm{CH}}_{3}\right)_{3} \mathrm{C}\right), 1.55-1.70\left(\mathrm{~m}, 2 \mathrm{H}, \mathrm{CH}_{2}\right), 2.05-2.15(\mathrm{~m}, 1 \mathrm{H}, \mathrm{CH}), 3.78(\mathrm{~s}, 3 \mathrm{H}$, $\left.\mathrm{OCH}_{3}\right), 5.04(\mathrm{dd}, J=5.8$ and $9.9 \mathrm{~Hz}, 1 \mathrm{H}, \mathrm{CHN}), 6.80-6.90(\mathrm{~m}, 2 \mathrm{H}, \mathrm{H}$ arom), 7.35-7.45 (m, $2 \mathrm{H}, \mathrm{H}$ arom). ${ }^{13} \mathbf{C}$ NMR $\left(\mathbf{C D C l}_{3}, 75.5 \mathbf{~ M H z}\right): \delta=22.0\left(\mathrm{CH}_{3}\right), 23.0\left(\mathrm{CH}_{3}\right), 24.8(\mathrm{CH}), 28.3$ $\left(\mathrm{C}\left(\underline{\mathrm{CH}}_{3}\right)_{3}\right), 40.5\left(\mathrm{CH}_{2}\right), 55.2(\mathrm{OMe}), 59.7(\mathrm{CHN}), 81.7\left(\underline{\mathrm{C}}\left(\mathrm{CH}_{3}\right)_{3}\right), 113.6(2 \mathrm{CH}$ arom $), 128.9$ (2 $\mathrm{CH}$ arom), 132.2 (C arom), 156.6 and 158.9 (C arom and $\mathrm{C}=\mathrm{O}$ ). LRMS (ESI): $\mathrm{m} / \mathrm{z}=309$ $\left[(\mathrm{M}+\mathrm{H})^{+}\right], 332\left[(\mathrm{M}+\mathrm{Na})^{+}\right], 348\left[(\mathrm{M}+\mathrm{K})^{+}\right]$. Anal. Calcd for $\mathbf{C}_{17} \mathbf{H}_{27} \mathbf{N O}_{4}: \mathbf{C}, 66.00 ; \mathrm{H}, 8.80 ; \mathrm{N}$, 4.53. Found: C, 65.67; H, 9.07; N, 4.28.

\section{- 1-[N-(tert-butoxycarbonyl)-N-(hydroxy)amino]-3-methyl-1-(tolyl)butane $6 \mathrm{~b}$}

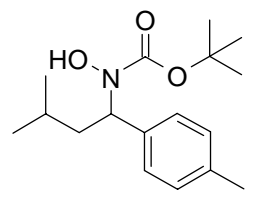

To a solution of sulfone 1a $(100 \mathrm{mg}, 0.29 \mathrm{mmol})$ in toluene $(3 \mathrm{~mL})$ and THF $(1 \mathrm{~mL})$ at room temperature under inert atmosphere, was added tolylmagnesium bromide $5 \mathbf{b}(0.45 \mathrm{M}$ solution in $\mathrm{Et}_{2} \mathrm{O}, 1.42 \mathrm{~mL}, 0.64 \mathrm{mmol}$ ). The reaction mixture was stirred at room temperature during 1 hour before being quenched by addition of a saturated aqueous solution of 
ammonium chloride. The resulting mixture was extracted twice by EtOAc. Combined organic layers were washed with brine, dried over anhydrous magnesium sulfate and the solvents were evaporated. The residue was purified by column chromatography on flash silica gel (Eluent: $\mathrm{CH}_{2} \mathrm{Cl}_{2}$ ). The product $\mathbf{6 b}$ was obtained as an oil $(69 \mathrm{mg}, 0.24 \mathrm{mmol})$.

Yield: 83\%. IR (film): 3206, 2955, 2923, 2869, 1687, 1513, 1471, 1456, 1396, 1367, 1258, 1175, 1138, $1093 \mathrm{~cm}^{-1} .{ }^{1} \mathbf{H}$ NMR (CDCl $\left.3,300 \mathbf{M H z}\right): \delta=0.95\left(\mathrm{~d}, J=6.4 \mathrm{~Hz}, 6 \mathrm{H}, 2 \mathrm{CH}_{3}\right)$, $1.43\left(\mathrm{~s}, 9 \mathrm{H},\left(\left(\underline{\mathrm{CH}}_{3}\right)_{3} \mathrm{C}\right), 1.55-1.70\left(\mathrm{~m}, 2 \mathrm{H}, \mathrm{CH}_{2}\right), 2.05-2.18(\mathrm{~m}, 1 \mathrm{H}, \mathrm{CH}), 2.31\left(\mathrm{~s}, 3 \mathrm{H}, \mathrm{CH}_{3}\right)\right.$, $5.06(\mathrm{dd}, J=5.8$ and $10.1 \mathrm{~Hz}, 1 \mathrm{H}, \mathrm{CHN}), 7.15-7.25$ (m, 2H, H arom), 7.20-7.30 (m, 2H, H arom). ${ }^{13} \mathbf{C}$ NMR (CDCl 3 , $\left.75.5 \mathrm{MHz}\right): \delta=21.0\left(\left(\underline{\mathrm{CH}}_{3}\right)_{3} \mathrm{C}\right), 21.9\left(\mathrm{CH}_{3}\right), 23.0\left(\mathrm{CH}_{3}\right), 24.8$ $(\mathrm{CH}), 28.3\left(\mathrm{C}\left(\underline{\mathrm{CH}}_{3}\right)_{3}\right), 40.4\left(\mathrm{CH}_{2}\right), 59.9(\mathrm{CHN}), 81.7\left(\underline{\mathrm{C}}\left(\mathrm{CH}_{3}\right)_{3}\right), 127.6(2 \mathrm{CH}$ arom $), 128.9$ (2 $\mathrm{CH}$ arom), 137.0 ( $\mathrm{C}$ arom), 137.1 ( $\mathrm{C}$ arom), $156.6(\mathrm{C}=\mathrm{O})$. LRMS (ESI): $\mathrm{m} / \mathrm{z}=316$ $\left[(\mathrm{M}+\mathrm{Na})^{+}\right], 609\left[(2 \mathrm{M}+\mathrm{Na})^{+}\right]$. Anal. Calcd for $\mathbf{C}_{17} \mathbf{H}_{27} \mathbf{N O}_{3}: \mathrm{C}, 69.60 ; \mathrm{H}, 9.28 ; \mathrm{N}, 4.78$. Found: C, 69.65; H, 9.36; N, 4.70.

- 1-[N-(tert-butoxycarbonyl)- $N$-(hydroxy)amino]-2-[ $N$-(tertbutoxycarbonyl)amino]-1-(4-methoxyphenyl)ethane 6c

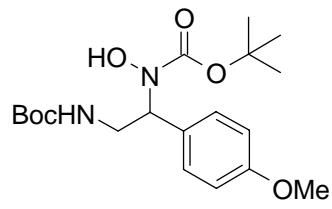

To a solution of sulfone $\mathbf{1 b}(100 \mathrm{mg}, 0.29 \mathrm{mmol})$ in toluene $(3 \mathrm{~mL})$ and THF $(0.5 \mathrm{~mL})$ at room temperature under inert atmosphere was added 4-methoxyphenylmagnesium bromide 5 a $(0.51 \mathrm{M}$ solution in toluene, $1.52 \mathrm{~mL}, 0.77 \mathrm{mmol})$ The reaction mixture was stirred at room temperature during 1 hour before being quenched by addition of a saturated aqueous solution of ammonium chloride. The resulting mixture was extracted twice by EtOAc. Combined organic layers were washed with brine, dried over anhydrous magnesium sulfate and the solvents were evaporated. The residue was purified by column chromatography on flash silica gel (Eluent: $\mathrm{CH}_{2} \mathrm{Cl}_{2}$ ). The product $\mathbf{6 c}$ was obtained as a white amorphous solid (61 $\mathrm{mg}, 0.16$ mmol).

Yield: 55\%. IR (film): 3341, 2977, 2936, 2839, 1696, 1513, 1366, 1292, 1247, 1175, 1116, $1037 \mathrm{~cm}^{-1} .{ }^{1} \mathbf{H}$ NMR $\left(\mathbf{C D C l}_{3}, 300 \mathbf{M H z}\right): \delta=1.44\left(\mathrm{~s}, 9 \mathrm{H},\left(\underline{\mathrm{CH}}_{3}\right)_{3} \mathrm{C}\right), 1.46\left(\mathrm{~s}, 9 \mathrm{H},\left(\underline{\mathrm{CH}}_{3}\right)_{3} \mathrm{C}\right)$, 3.13-3.35 (m, $1 \mathrm{H}, \mathrm{H}$ of $\left.\mathrm{CH}_{2} \mathrm{~N}\right), 3.79\left(\mathrm{~s}, 3 \mathrm{H}, \mathrm{OCH}_{3}\right), 3.85-4.02\left(\mathrm{~m}, 1 \mathrm{H}, \mathrm{H}\right.$ of $\left.\mathrm{CH}_{2} \mathrm{~N}\right), 5.01$ (br s, 1H, NHBoc), 5.07 (dd, $J=3.9$ and $11.4 \mathrm{~Hz}, 1 \mathrm{H}, \mathrm{CHN}), 6.85$ (d, $J=8.7 \mathrm{~Hz}, 2 \mathrm{H}, \mathrm{H}$ arom), 7.34 (d, $J=8.7 \mathrm{~Hz}, 2 \mathrm{H}, \mathrm{H}$ arom), 7.82 (br s, $1 \mathrm{H}, \mathrm{OH}) .{ }^{13} \mathbf{C} \mathbf{~ N M R}\left(\mathbf{C D C l}_{3}, \mathbf{7 5 . 5} \mathbf{M H z}\right): \delta=28.3$ (2 $\left.\mathrm{C}\left(\underline{\mathrm{CH}}_{3}\right)_{3}\right), 41.5\left(\mathrm{CH}_{2} \mathrm{~N}\right), 55.2\left(\mathrm{CH}_{3} \mathrm{O}\right), 61.4(\mathrm{CHN}), 80.6\left(\underline{\mathrm{C}}\left(\mathrm{CH}_{3}\right)_{3}\right), 81.1\left(\underline{\mathrm{C}}\left(\mathrm{CH}_{3}\right)_{3}\right), 113.8(2$ $\mathrm{CH}$ arom), 128.8 (2 $\mathrm{CH}$ arom), $129.7(\mathrm{C}$ arom), $159.2(\mathrm{C}=\mathrm{O})$. LRMS (ESI): $\mathrm{m} / \mathrm{z}=383$ 
$\left[(\mathrm{M}+\mathrm{H})^{+}\right], 405\left[(\mathrm{M}+\mathrm{Na})^{+}\right], 787\left[(2 \mathrm{M}+\mathrm{Na})^{+}\right]$. Anal. Calcd for $\mathbf{C}_{\mathbf{1 9}} \mathbf{H}_{\mathbf{3 0}} \mathbf{N}_{\mathbf{2}} \mathbf{O}_{\mathbf{6}}: \mathrm{C}, 59.67 ; \mathrm{H}, 7.91$; N, 7.33. Found: C, 59.58; H, 8.20; N, 7.11.

- 1-[N-(tert-butoxycarbonyl)- $N$-(hydroxy)amino]-2-[N-(tertbutoxycarbonyl)amino]-1-(tolyl)ethane 6d

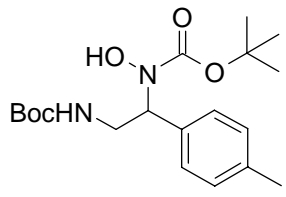

To a solution of sulfone $\mathbf{1 b}(100 \mathrm{mg}, 0.24 \mathrm{mmol})$ in toluene $(3 \mathrm{~mL})$ and THF $(0.5 \mathrm{~mL})$ at room temperature under inert atmosphere was added tolylmagnesium bromide $5 \mathbf{b}(0.45 \mathrm{M}$ solution in $\mathrm{Et}_{2} \mathrm{O}, 1.71 \mathrm{~mL}, 0.77 \mathrm{mmol}$ ). The reaction mixture was stirred at room temperature during 1 hour before being quenched by addition of a saturated aqueous solution of ammonium chloride. The resulting mixture was extracted twice by EtOAc. Combined organic layers were washed with brine, dried over anhydrous magnesium sulfate and the solvents were evaporated. The residue was purified by column chromatography on flash silica gel (Eluent: $\mathrm{CH}_{2} \mathrm{Cl}_{2}$ ). The product $\mathbf{6 d}$ was obtained as a white amorphous solid (54 mg, 0.15 mmol).

Yield: 63\%. IR (film): 3315, 2977, 2936, 2865, 1700, 1674, 1516, 1393, 1367, 1295, 1254, 1171, $1112 \mathrm{~cm}^{-1} .{ }^{\mathbf{1}} \mathbf{H}$ NMR $\left(\mathbf{C D C l}_{3}, 300 \mathbf{M H z}\right): \delta=1.44\left(\mathrm{~s}, 9 \mathrm{H},\left(\underline{\mathrm{CH}}_{3}\right)_{3} \mathrm{C}\right), 1.46(\mathrm{~s}, 9 \mathrm{H}$, $\left.\left(\underline{\mathrm{CH}}_{3}\right)_{3} \mathrm{C}\right), 2.32\left(\mathrm{~s}, 3 \mathrm{H}, \mathrm{CH}_{3}\right), 3.15-3.28\left(\mathrm{~m}, 1 \mathrm{H}, \mathrm{H}\right.$ of $\left.\mathrm{CH}_{2} \mathrm{~N}\right), 3.80-4.00\left(\mathrm{~m}, 1 \mathrm{H}, \mathrm{H}\right.$ of $\left.\mathrm{CH}_{2} \mathrm{~N}\right)$, 5.01 (br s, 1H, NHBoc), 5.09 (dd, $J=3.7$ and $11.4 \mathrm{~Hz}, 1 \mathrm{H}, \mathrm{CHN}), 7.13$ (d, J=7.9 Hz, 2H, H arom), 7.30 (d, $J=7.9 \mathrm{~Hz}, 2 \mathrm{H}, \mathrm{H}$ arom), 7.84 (s, 1H, OH). ${ }^{13} \mathbf{C} \mathbf{N M R}\left(\mathbf{C D C l}_{3}, \mathbf{7 5 . 5} \mathbf{~ M H z}\right): \delta=$ $21.1\left(\mathrm{CH}_{3}\right), 26.9\left(\mathrm{CH}_{2}\right), 28.4\left(2 \mathrm{C}\left(\underline{\mathrm{CH}}_{3}\right)_{3}\right), 41.5\left(\mathrm{CH}_{2} \mathrm{~N}\right), 61.8(\mathrm{CHN}), 80.6\left(\underline{\mathrm{C}}\left(\mathrm{CH}_{3}\right)_{3}\right), 81.2$ $\left(\underline{\mathrm{C}}\left(\mathrm{CH}_{3}\right)_{3}\right), 127.4(2 \mathrm{CH}$ arom), $129.1(2 \mathrm{CH}$ arom), $134.6(\mathrm{C}$ arom $), 137.5(\mathrm{C}$ arom $), 156.5$ $(\mathrm{C}=\mathrm{O}), 157.8(\mathrm{C}=\mathrm{O})$. LRMS (ESI): $\mathrm{m} / \mathrm{z}=389\left[(\mathrm{M}+\mathrm{Na})^{+}\right], 755\left[(2 \mathrm{M}+\mathrm{Na})^{+}\right]$. Anal. Calcd for $\mathbf{C}_{19} \mathbf{H}_{30} \mathbf{N}_{2} \mathbf{O}_{5}$ : C, 62.28; H, 8.26; N, 7.65. Found: C, 62.30; H, 8.58; N, 7.40. 


\section{Preparation of benzothien-2-yl $N$-hydroxylamine $7 \mathrm{~b}$}

- 1-[N-(tert-butoxycarbonyl)- $N$-(hydroxy)amino]-2-[N-(tertbutoxycarbonyl)amino]-1-(benzothien-2-yl)ethane $7 \mathrm{~b}$

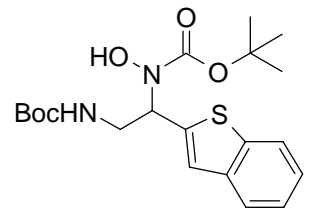

To a solution of thianaphtene $(319 \mathrm{mg}, 2.38 \mathrm{mmol})$ in ether $(2 \mathrm{~mL})$ at room temperature was added $n$-BuLi (1.28 $\mathrm{M}$ in hexane, $1.86 \mathrm{~mL}, 2.38 \mathrm{mmol})$. After $55 \mathrm{~min}$ of reaction, $\mathrm{MgBr}_{2}$. OEt ${ }_{2}$ (614 mg, $2.38 \mathrm{mmol}$ ) was added in three portions to give a suspension of 2benzothienylmagnesium bromide. To this solution was added a solution of sulfone 1b (300 $\mathrm{mg}, 0.72 \mathrm{mmol})$ in toluene $(5 \mathrm{~mL})$ and THF $(1 \mathrm{~mL})$. The resulting mixture was allowed to stir at room temperature during $1 \mathrm{~h}$. It was then quenched by addition of a saturated aqueous solution of ammonium chloride. Aqueous layer was extracted twice by EtOAc. Combined organic layers were washed by brine, dried over anhydrous magnesium sulfate and filtered. The solvents were removed under reduced pressure and the residue was purified by column chromatography on flash silica gel (Eluent: $\mathrm{CH}_{2} \mathrm{Cl}_{2}$ ). The product $\mathbf{7 b}$ was obtained as a pale amorphous solid (84 mg, $0.21 \mathrm{mmol}$ ).

Yield: 29\%. IR (neat): 3337, 2974, 2932, 1700, 1677, 1520, 1393, 1366, 1292, 1250, 1172, $1119 \mathrm{~cm}^{-1} .{ }^{1} \mathbf{H}$ NMR $\left(\mathbf{C D C l}_{3}, 300 \mathrm{MHz}\right): \delta=1.47\left(\mathrm{~s}, 9 \mathrm{H},\left(\underline{\mathrm{CH}}_{3}\right)_{3} \mathrm{C}\right), 3.30-3.45(\mathrm{~m}, 1 \mathrm{H}, \mathrm{H}$ of $\left.\mathrm{CH}_{2} \mathrm{~N}\right), 3.90-4.05\left(\mathrm{~m}, 1 \mathrm{H}, \mathrm{H}\right.$ of $\left.\mathrm{CH}_{2} \mathrm{~N}\right), 5.15(\mathrm{t}, J=7.2 \mathrm{~Hz}, 1 \mathrm{H}, \mathrm{NHBoc}), 5.43(\mathrm{dd}, J=3.9$ and $11.1 \mathrm{~Hz}, 1 \mathrm{H}, \mathrm{CHN}), 7.24-7.36$ (m, 3H, H arom), 7.68-7.74 (m, 1H, H arom), 7.74-7.80 (m, $1 \mathrm{H}, \mathrm{H}$ arom). ${ }^{13} \mathbf{C}$ NMR $\left(\mathbf{C D C l}_{3}, 75.5 \mathbf{M H z}\right): \delta=28.3\left(\left(\underline{\mathrm{CH}}_{3}\right)_{3} \mathrm{C}\right), 41.9\left(\mathrm{CH}_{2} \mathrm{~N}\right), 80.8$ $\left(\underline{\mathrm{C}}\left(\mathrm{CH}_{3}\right)_{3}\right), 81.7\left(\underline{\mathrm{C}}\left(\mathrm{CH}_{3}\right)_{3}\right), 122.2(\mathrm{CH}$ arom $), 122.3(\mathrm{CH}$ arom $), 123.5(\mathrm{CH}$ arom $), 124.2(\mathrm{CH}$ arom), $124.3(\mathrm{CH}$ arom), 139.2 (C arom), 139.6 (C arom), $140.0(\mathrm{C}$ arom), $156.4(\mathrm{C}=\mathrm{O})$, $157.8(\mathrm{C}=\mathrm{O})$. LRMS (ESI): $\mathrm{m} / \mathrm{z}=409\left[(\mathrm{M}+\mathrm{H})^{+}\right], 431\left[(\mathrm{M}+\mathrm{Na})^{+}\right], 817\left[(2 \mathrm{M}+\mathrm{H})^{+}\right], 839$ $\left[(2 \mathrm{M}+\mathrm{Na})^{+}\right]$. Anal. Calcd for $\mathbf{C}_{20} \mathbf{H}_{28} \mathbf{N}_{2} \mathbf{O}_{5} \mathrm{~S}: \mathrm{C}, 58.81 ; \mathrm{H}, 6.91 ; \mathrm{N}, 6.86$. Found: $\mathrm{C}, 58.84 ; \mathrm{H}$, $7.29 ; \mathrm{N}, 6.56$. 


\section{Preparation of indolic $N$-hydroxylamines 11}

\section{General procedure}

To a solution of the indolic core in THF at $-78{ }^{\circ} \mathrm{C}$ under inert atmosphere was added methylmagnesium bromide $\left(3 \mathrm{M}\right.$ solution in $\left.\mathrm{Et}_{2} \mathrm{O}\right)$ and the resulting solution was stirred during $15 \mathrm{~min}$. A solution of the sulfone $\mathbf{1 b}$ dissolved into THF was slowly added and the reaction was allowed to reach $-5{ }^{\circ} \mathrm{C}$ over a few hours and was stirred overnight. It was then quenched by addition of an aqueous saturated solution of ammonium chloride. The crude mixture was extracted three times by EtOAc. Organic layers were washed by an aqueous saturated solution of sodium chloride and then dried over anhydrous magnesium sulfate. After the removal of the solvent, the crude mixture was purified by column chromatography on silica gel.

- 1-[N-(tert-butoxycarbonyl)- $N$-(hydroxy)amino]-2-[ $N$-(tertbutoxycarbonyl)amino]-1-(5'-bromoindol-3'-yl)ethane 11b

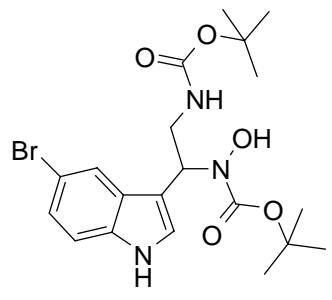

Prepared according to the above general procedure, by reaction of $150 \mathrm{mg}$ of sulfone 1b $(0.36 \mathrm{mmol})$ with the 5-bromoindolylmagnesium bromide prepared from $212 \mathrm{mg}$ of 5bromoindole $(1.08 \mathrm{mmol})$ and $0.36 \mathrm{~mL}$ of $\mathrm{MeMgBr}(1.08 \mathrm{mmol})$ in $3 \mathrm{~mL}$ of THF. After purification by column chromatography on silica gel (Eluent: $\mathrm{CH}_{2} \mathrm{Cl}_{2}$ then EtOAc), the product 11b was obtained as a white solid (137 $\mathrm{mg}, 0.29 \mathrm{mmol})$.

Yield: 81\%. IR (film): 3324, 2972, 2914, 2846, 1684, 1519, 1461, 1392, 1367, 1287, 1255, $1167 \mathrm{~cm}^{-1} .{ }^{1} \mathbf{H}$ NMR $\left(\mathbf{C D C l}_{3}, 300 \mathbf{M H z}\right): \delta=1.46\left(\mathrm{~s}, 18 \mathrm{H}, \mathrm{C}\left(\underline{\mathrm{CH}}_{3}\right)_{3}\right), 3.23(\mathrm{dt}, J=4.7$ and $14.7 \mathrm{~Hz}, 1 \mathrm{H}, \mathrm{CH}$ of $\left.\mathrm{CH}_{2} \mathrm{~N}\right), 3.77-3.95\left(\mathrm{~m}, 1 \mathrm{H}, \mathrm{CH}\right.$ of $\left.\mathrm{CH}_{2} \mathrm{~N}\right), 5.18-5.28(\mathrm{~m}, 1 \mathrm{H}, \mathrm{NHBoc})$, $5.38(\mathrm{dd}, J=3.8$ and $11.3 \mathrm{~Hz}, 1 \mathrm{H}, \mathrm{CHN}), 7.10(\mathrm{dt}, J=1.3$ and $7.8 \mathrm{~Hz}, 1 \mathrm{H}, \mathrm{H}$ indol), 7.08-7.19 (m, 2H, H indol), 7.78 (d, $J=1 \mathrm{~Hz}, 1 \mathrm{H}, \mathrm{CH}$ indol), 8.05 (s, 1H, OH), 8.91 (s, 1H, NH indol).

${ }^{13} \mathbf{C}$ NMR (CDCl $\left.3,75.5 \mathrm{MHz}\right): \delta=28.3\left(2 \mathrm{C}\left(\underline{\mathrm{CH}}_{3}\right)_{3}\right), 41.1\left(\mathrm{CH}_{2} \mathrm{~N}\right), 55.1(\mathrm{CHN}), 80.6$ $\left(\underline{\mathrm{C}}\left(\mathrm{CH}_{3}\right)_{3}\right), 81.6\left(\underline{\mathrm{C}}\left(\mathrm{CH}_{3}\right)_{3}\right), 112.3(\mathrm{C}$ indol $), 112.8(\mathrm{C}$ indol $), 112.8(\mathrm{CH}$ indol $), 121.5(\mathrm{CH}$ indol), $124.4(\mathrm{CH}$ indol), $124.7(\mathrm{CH}$ indol), $128.0(\mathrm{C}$ indol), $134.4(\mathrm{C}$ indol $), 156.6(\mathrm{C}=\mathrm{O})$, $158.0(\mathrm{C}=\mathrm{O})$. LRMS (ESI): $\mathrm{m} / \mathrm{z}=476$ and $478\left[(\mathrm{M}+\mathrm{Li})^{+}\right], 492$ and $494\left[(\mathrm{M}+\mathrm{Na})^{+}\right], 947$ and 949 [(dimer+Li $\left.)^{+}\right], 963$ and $965\left[(\operatorname{dimer}+\mathrm{Na})^{+}\right]$. Anal. Calcd for $\mathbf{C}_{20} \mathbf{H}_{28} \mathbf{B r N}_{3} \mathbf{O}_{5}: \mathbf{C}, 51.08 ; \mathrm{H}$, 6.00; N, 8.94. Found: C, 50.87; H, 6.13; N, 8.73. 
- $\quad$ 1-[ $N$-(tert-butoxycarbonyl)- $N$-(hydroxy)amino]-2-[ $N$-(tertbutoxycarbonyl)amino]-1-(5'-chloroindol-3'-yl)ethane 11c

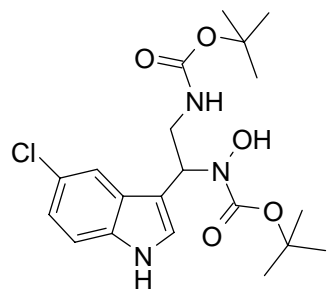

Prepared according to the above procedure, by reaction of $100 \mathrm{mg}$ of sulfone $\mathbf{1 b}(0.24$ mmol) with the 5-chloroindolylmagnesium bromide prepared from $109 \mathrm{mg}$ of 5-chloroindole $(0.72 \mathrm{mmol})$ and $0.24 \mathrm{~mL}$ of $\mathrm{MeMgBr}(0.72 \mathrm{mmol})$ in $3 \mathrm{~mL}$ of THF. After purification by column chromatography on silica gel (Eluent: $\mathrm{Et}_{2} \mathrm{O} /$ pentane, 4:1), the product 11c was obtained as a colorless solid $(81 \mathrm{mg}, 0.19 \mathrm{mmol})$.

Yield: 79\%. IR (film): 3322, 2981, 2932, 1692, 1516, 1464, 1374, 1288, 1250, 1164, 1116 $\mathrm{cm}^{-1} .{ }^{1} \mathbf{H}$ NMR (CDCl $\left.3,300 \mathbf{~ M H z}\right): \delta=1.47\left(\mathrm{~s}, 9 \mathrm{H}, \mathrm{C}\left(\underline{\mathrm{CH}}_{3}\right)_{3}\right), 1.48\left(\mathrm{~s}, 9 \mathrm{H}, \mathrm{C}\left(\underline{\mathrm{CH}}_{3}\right)_{3}\right), 3.21-$ $3.29\left(\mathrm{~m}, 1 \mathrm{H}, \mathrm{CH}\right.$ of $\left.\mathrm{CH}_{2} \mathrm{~N}\right), 3.85-4.02\left(\mathrm{~m}, 1 \mathrm{H}, \mathrm{CH}\right.$ of $\left.\mathrm{CH}_{2} \mathrm{~N}\right), 5.10(\mathrm{t}, J=7.7 \mathrm{~Hz}, 1 \mathrm{H}, \mathrm{NHBoc})$, $5.41(\mathrm{dd}, J=3.9$ and $11.4 \mathrm{~Hz}, 1 \mathrm{H}, \mathrm{CHN}), 7.09$ (dd, $J=1.8$ and $8.4 \mathrm{~Hz}, 1 \mathrm{H}, \mathrm{H}$ indol), 7.18-7.26 (m, 2H, H indol), 7.66 (d, J=1.8 Hz, 1H, CH indol), 7.82 (br s, 1H, OH), $8.57(\mathrm{~s}, 1 \mathrm{H}, \mathrm{NH}$ indol). ${ }^{13} \mathrm{C}$ NMR (CDCl 3 , $\left.75.5 \mathrm{MHz}\right): \delta=28.3\left(2 \mathrm{C}\left(\underline{\mathrm{CH}}_{3}\right)_{3}\right), 41.1\left(\mathrm{CH}_{2} \mathrm{~N}\right), 55.1(\mathrm{CHN}), 80.6$ $\left(\underline{\mathrm{C}}\left(\mathrm{CH}_{3}\right)_{3}\right), 81.6\left(\underline{\mathrm{C}}\left(\mathrm{CH}_{3}\right)_{3}\right), 111.4(\mathrm{C}$ indol $), 112.4(\mathrm{CH}$ indol $), 118.4(\mathrm{CH}$ indol $), 122.2(\mathrm{CH}$ indol), $124.6(\mathrm{CH}$ indol), 125.2 ( $\mathrm{C}$ indol), $127.30(\mathrm{C}$ indol), $134.1(\mathrm{C}$ indol), $156.6(\mathrm{C}=\mathrm{O})$, $158.0(\mathrm{C}=\mathrm{O})$. LRMS (ESI): $\mathrm{m} / \mathrm{z}=425\left[(\mathrm{M}+\mathrm{H})^{+}\right]$. Anal. Calcd for $\mathbf{C}_{20} \mathbf{H}_{28} \mathbf{C l N}_{3} \mathbf{O}_{5}$ : C, 56.40; H, 6.63; N, 9.87. Found: C, 56.21; H, 6.55; N, 9.39.

- 1-[N-(tert-butoxycarbonyl)- $N$-(hydroxy)amino]-2-[ $N$-(tertbutoxycarbonyl)amino]-1-(5'-fluoroindol-3'-yl)ethane 11d

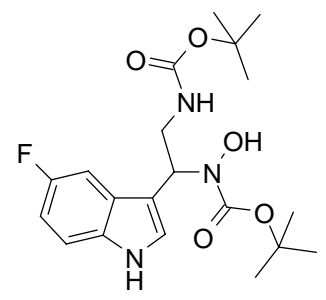

Prepared according to the above general procedure, by reaction of $100 \mathrm{mg}$ of sulfone 1b $(0.24 \mathrm{mmol})$ with the 5-fluoroindolylmagnesium bromide prepared from $98 \mathrm{mg}$ of 5fluoroindole $(0.72 \mathrm{mmol})$ and $0.24 \mathrm{~mL}$ of $\mathrm{MeMgBr}(0.72 \mathrm{mmol})$ in $3 \mathrm{~mL}$ of THF. After purification by column chromatography on silica gel (Eluent: $\mathrm{Et}_{2} \mathrm{O} /$ pentane, 2:3), the product 11d was obtained as a colorless solid (90 mg, $0.22 \mathrm{mmol}$ ). 
Yield: 92\%. ${ }^{\mathbf{1}} \mathbf{H}$ NMR (CDCl $\left.3,300 \mathbf{M H z}\right): \delta=1.38\left(\mathrm{~s}, 9 \mathrm{H}, \mathrm{C}\left(\underline{\mathrm{CH}}_{3}\right)_{3}\right), 1.39$ (s, 9H, C( $\left.\left.\underline{\mathrm{CH}}_{3}\right)_{3}\right)$, 3.05-3.25 (m, 1H, CH of $\left.\mathrm{CH}_{2} \mathrm{~N}\right), 3.70-3.90\left(\mathrm{~m}, 1 \mathrm{H}, \mathrm{CH}\right.$ of $\left.\mathrm{CH}_{2} \mathrm{~N}\right), 5.15$ (br s, 1H, NHBoc), $5.33(\mathrm{dd}, J=3.6$ and $11.1 \mathrm{~Hz}, 1 \mathrm{H}, \mathrm{CHN}), 6.78(\mathrm{dt}, J=2.4$ and $9.0 \mathrm{~Hz}, 1 \mathrm{H}, \mathrm{H}$ indol), 7.05-7.15 (m, 2H, $\mathrm{H}$ indol), 7.24 (dd, $J=2.4$ and $9.9 \mathrm{~Hz}, 1 \mathrm{H}, \mathrm{CH}$ indol), 8.0 (br s, $1 \mathrm{H}, \mathrm{OH}), 8.72(\mathrm{~s}, 1 \mathrm{H}$, $\mathrm{NH}$ indol). ${ }^{13} \mathbf{C}$ NMR (CDCl 3 , $\left.75.5 \mathbf{M H z}\right): \delta=28.3\left(2 \mathrm{C}\left(\underline{\mathrm{CH}}_{3}\right)_{3}\right), 41.1\left(\mathrm{CH}_{2} \mathrm{~N}\right), 55.1(\mathrm{CHN})$, $80.6\left(\underline{\mathrm{C}}\left(\mathrm{CH}_{3}\right)_{3}\right), 81.5\left(\underline{\mathrm{C}}\left(\mathrm{CH}_{3}\right)_{3}\right), 103.8$ and $104.1(\mathrm{CH}$ indol), 110.2 and $110.5(\mathrm{CH}$ indol), 111.9 and 112.0 ( $\mathrm{CH}$ indol), 111.9 (C indol), 124.9 (CH indol), 126.6 and 126.7 (C indol), 132.3 (C indol), 156.3 (C indol), $158.0(\mathrm{C}=\mathrm{O}), 159.4(\mathrm{C}=\mathrm{O})$. LRMS (ESI): $\mathrm{m} / \mathrm{z}=409$ $\left[(\mathrm{M}+\mathrm{H})^{+}\right], 432\left[(\mathrm{M}+\mathrm{Na})^{+}\right]$. HRMS (ESI) calcd $\mathbf{C}_{20} \mathbf{H}_{28} \mathbf{N}_{3} \mathbf{O}_{5} \mathbf{F N a}\left[(\mathrm{M}+\mathrm{Na})^{+}\right]: 432.1911$. Found: 432.1901.

- 1-[N-(tert-butoxycarbonyl)- $N$-(hydroxy)amino]-2-[ $N$-(tertbutoxycarbonyl)amino]-1-(6'-bromoindol-3'-yl)ethane 11e

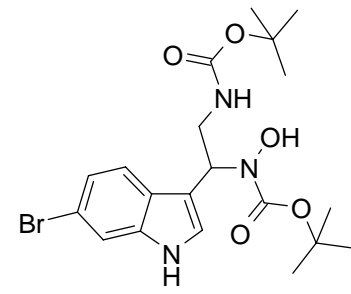

Prepared according to the above general procedure, by reaction of $300 \mathrm{mg}$ of sulfone 1b $(0.72 \mathrm{mmol})$ with the 6-bromoindolylmagnesium bromide prepared from $424 \mathrm{mg}$ of 6bromoindole $(2.16 \mathrm{mmol})$ and $0.72 \mathrm{~mL}$ of $\mathrm{MeMgBr}(2.16 \mathrm{mmol})$ in $7 \mathrm{~mL}$ of THF. After purification by column chromatography on silica gel (Eluent: $\mathrm{Et}_{2} \mathrm{O} /$ pentane, 1:1), the product 11e was obtained as a beige solid $(127 \mathrm{mg}, 0.27 \mathrm{mmol})$.

Yield: 38\%. IR (film): 3340, 2981, 2929, 1696, 1520, 1460, 1396, 1370, 1292, 1254, 1172 , $1116 \mathrm{~cm}^{-1} .{ }^{1} \mathbf{H}$ NMR (CDCl $\left.3,300 \mathbf{M H z}\right): \delta=1.45\left(\mathrm{~s}, 9 \mathrm{H}, \mathrm{C}\left(\underline{\mathrm{CH}}_{3}\right)_{3}\right), 1.48\left(\mathrm{~s}, 9 \mathrm{H}, \mathrm{C}\left(\underline{\mathrm{CH}}_{3}\right)_{3}\right)$, $3.26\left(\mathrm{dt}, J=4.7\right.$ and $12.3 \mathrm{~Hz}, 1 \mathrm{H}, \mathrm{CH}$ of $\left.\mathrm{CH}_{2} \mathrm{~N}\right), 3.85-4.05\left(\mathrm{~m}, 1 \mathrm{H}, \mathrm{CH}\right.$ of $\left.\mathrm{CH}_{2} \mathrm{~N}\right), 5.05-5.15$ (m, $1 \mathrm{H}, \mathrm{NHBoc}), 5.38(\mathrm{dd}, J=3.9$ and $11.5 \mathrm{~Hz}, 1 \mathrm{H}, \mathrm{CHN}), 7.20(\mathrm{dd}, J=1.8$ and $8.5 \mathrm{~Hz}, 1 \mathrm{H}$, $\mathrm{H}$ indol), 7.26 (s, 1H, H indol), 7.49 (d, $J=1.5 \mathrm{~Hz}, 1 \mathrm{H}, \mathrm{CH}$ indol), 7.56 (d, $J=8.5 \mathrm{~Hz}, 1 \mathrm{H}, \mathrm{H}$ indol), 7.83 (br s, 1H, OH), 8.41 (s, 1H, NH indol). ${ }^{13} \mathbf{C}$ NMR (CDCl 3 , $75.5 \mathbf{~ M H z ) : ~} \delta=28.3$ $\left(\mathrm{C}\left(\underline{\mathrm{CH}}_{3}\right)_{3}\right), 28.4\left(\mathrm{C}\left(\underline{\mathrm{CH}}_{3}\right)_{3}\right), 41.2\left(\mathrm{CH}_{2} \mathrm{~N}\right), 54.9(\mathrm{CHN}), 80.5\left(\underline{\mathrm{C}}\left(\mathrm{CH}_{3}\right)_{3}\right), 81.4\left(\underline{\mathrm{C}}\left(\mathrm{CH}_{3}\right)_{3}\right), 112.0$ (C indol), 114.2 (CH indol), $115.4(\mathrm{C}$ indol), $120.2(\mathrm{CH}$ indol), $122.7(\mathrm{CH}$ indol), $123.8(\mathrm{CH}$ indol), 125.2 (C indol), 136.5 ( $\mathrm{C}$ indol), $156.7(\mathrm{C}=\mathrm{O}), 157.9(\mathrm{C}=\mathrm{O})$. LRMS (ESI): $\mathrm{m} / \mathrm{z}=492$ and $494\left[(\mathrm{M}+\mathrm{Na})^{+}\right]$. HRMS (ESI) calcd for $\mathbf{C}_{20} \mathbf{H}_{30} \mathbf{N}_{3} \mathbf{O}_{5}{ }^{\mathbf{} 1} \mathbf{B r N a}\left[(\mathrm{M}+\mathrm{Na})^{+}\right]$: 494.1267 . Found: 494.1260 . 


\section{Preparation of indolic 1,2-diamines 12}

- 1-[N-(tert-butoxycarbonyl)amino]-2-[ $N$-(tert-butoxycarbonyl)amino]-1-(indol-3'yl)ethane 12a

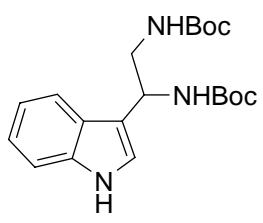

$N$-Hydroxylamine 11b (200 $\mathrm{mg}, 0.51 \mathrm{mmol})$ was dissolved into $3 \mathrm{~mL}$ of anhydrous THF under inert atmosphere. 12 equiv of ungazed water $(0.11 \mathrm{~mL}, 6.12 \mathrm{mmol})$ were added, immediately followed by 3 equiv of a $\mathrm{SmI}_{2}$ solution in THF $(1.53 \mathrm{mmol}, 15.3 \mathrm{~mL})$. The reaction was allowed to stir at room temperature during one hour. It was then quenched by air and then by the addition of a saturated aqueous solution of sodium thiosulfate. The resulting aqueous mixture was extracted three times by EtOAc. Combined organic layers were washed with brine and dried over anhydrous magnesium sulfate. After the removal of the solvent, the residue was purified by column chromatography (Eluent: $\mathrm{Et}_{2} \mathrm{O} /$ pentane, 1:1). The amine 12a was obtained as a colorless solid (123 $\mathrm{mg}, 0.33 \mathrm{mmol})$.

Yield: 65 \%. IR (film): 3341, 3060, 2977, 2936, 1692, 1513, 1393, 1367, 1245, $1172 \mathrm{~cm}^{-1}$. ${ }^{1} \mathbf{H}$ NMR (CDCl $\left.3,300 \mathrm{MHz}\right): \delta=1.45\left(\mathrm{~s}, 18 \mathrm{H}, \mathrm{C}\left(\underline{\mathrm{CH}}_{3}\right)_{3}\right), 3.45-3.80\left(\mathrm{~m}, 2 \mathrm{H}, \mathrm{CH}_{2} \mathrm{~N}\right), 5.00-$ $5.20(\mathrm{~m}, 3 \mathrm{H}, 2 \mathrm{NHBoc}$ and $\mathrm{CHN}), 6.98(\mathrm{~s}, 1 \mathrm{H}, \mathrm{H}$ indol), $7.09(\mathrm{dt}, J=1.2$ and $8.1 \mathrm{~Hz}, 1 \mathrm{H}, \mathrm{H}$ indol), 7.14 (dt, $J=1.2$ and $7.2 \mathrm{~Hz}, 1 \mathrm{H}, \mathrm{H}$ indol), 7.33 (d, $J=7.8 \mathrm{~Hz}, 1 \mathrm{H}, \mathrm{H}$ indol), 7.62 (d, $J=$ $8.1 \mathrm{~Hz}, 1 \mathrm{H}, \mathrm{CH}$ indol), 8.80 (s, $1 \mathrm{H}, \mathrm{NH}$ indol). ${ }^{13} \mathbf{C} \mathbf{~ N M R}\left(\mathbf{C D C l}_{3}, 75.5 \mathbf{M H z}\right): \delta=28.4(2$ $\left.\mathrm{C}\left(\underline{\mathrm{CH}}_{3}\right)_{3}\right), 45.0\left(\mathrm{CH}_{2} \mathrm{~N}\right), 48.5(\mathrm{CHN}), 79.4\left(\underline{\mathrm{C}}\left(\mathrm{CH}_{3}\right)_{3}\right), 79.5\left(\underline{\mathrm{C}}\left(\mathrm{CH}_{3}\right)_{3}\right), 111.5(\mathrm{CH}$ indol$)$, 114.4 (C indol), 119.0 ( $\mathrm{CH}$ indol), 119.6 ( $\mathrm{CH}$ indol), 121.7 ( $\mathrm{CH}$ indol), 122.2 (CH indol), $125.7(\mathrm{C}$ indol), $136.5(\mathrm{C}$ indol), $156.0(\mathrm{C}=\mathrm{O}), 156.6(\mathrm{C}=\mathrm{O})$. LRMS (ESI): $\mathrm{m} / \mathrm{z}=398$ $\left[(\mathrm{M}+\mathrm{Na})^{+}\right], 414\left[(\mathrm{M}+\mathrm{K})^{+}\right]$. HRMS (ESI) calcd for $\mathbf{C}_{20} \mathbf{H}_{29} \mathbf{N}_{3} \mathbf{O}_{4} \mathbf{N a}\left[(\mathrm{M}+\mathrm{Na})^{+}\right]: 398.2050$. Found: 398.2057.

- 1-[N-(tert-butoxycarbonyl)amino]-2-[ $N$-(tert-butoxycarbonyl)amino]-1-(5'bromoindol-3'-yl)ethane 12b

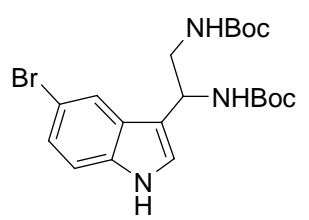

$\mathrm{N}$-Hydroxylamine 11c (423 $\mathrm{mg}, 0.90 \mathrm{mmol})$ was dissolved into $5 \mathrm{~mL}$ of anhydrous THF under inert atmosphere. 16 equiv of ungazed water $(0.26 \mathrm{~mL}, 14.4 \mathrm{mmol})$ were added, 
immediately followed by 4 equiv of a $\mathrm{SmI}_{2}$ solution in THF $(3.6 \mathrm{mmol}, 36 \mathrm{~mL})$. The reaction was allowed to stir at room temperature during one hour. It was then quenched by air and then by the addition of a saturated aqueous solution of sodium thiosulfate. The resulting aqueous mixture was extracted three times by EtOAc. Combined organic layers were washed with brine and dried over anhydrous magnesium sulfate. After the removal of the solvent, the residue was purified by column chromatography (Eluent: $\mathrm{Et}_{2} \mathrm{O}$ /pentane, 1:1). The amine 12b was obtained as a colorless solid (332 $\mathrm{mg}, 0.73 \mathrm{mmol})$.

Yield: 81\%. IR (film): 3313, 2978, 2932, 2874, 1694, 1513, 1455, 1394, 1369, 1251, 1166 $\mathrm{cm}^{-1} .{ }^{1} \mathbf{H}$ NMR (CDCl $\left.3,300 \mathbf{M H z}\right): \delta=1.45$ (s, 9H, C( $\left.\left.\underline{\mathrm{CH}}_{3}\right)_{3}\right), 1.46\left(\mathrm{~s}, 9 \mathrm{H}, \mathrm{C}\left(\underline{\mathrm{CH}}_{3}\right)_{3}\right), 3.45-$ $3.70\left(\mathrm{~m}, 2 \mathrm{H}, \mathrm{CH}_{2} \mathrm{~N}\right), 4.90-5.10$ (m, 2H, $2 \mathrm{NHBoc}$ ), 5.10-5.20 (br s, 1H, CHN), 6.98 (s, 1H, H indol), 7.21 (d, $J=8.7 \mathrm{~Hz}, 1 \mathrm{H}, \mathrm{H}$ indol), 7.24 (dd, $J=2.3$ and $9.3 \mathrm{~Hz}, 1 \mathrm{H}, \mathrm{H}$ indol), 7.73 (d, $J=$ $1.7 \mathrm{~Hz}, 1 \mathrm{H}, \mathrm{CH}$ indol), 8.76 (s, $1 \mathrm{H}, \mathrm{N}$ indol). ${ }^{13} \mathbf{C} \mathbf{~ N M R}\left(\mathbf{C D C l}_{3}, \mathbf{7 5 . 5} \mathbf{M H z}\right): \delta=28.4$ $\left(\mathrm{C}\left(\underline{\mathrm{CH}}_{3}\right)_{3}\right), 29.0\left(\mathrm{C}\left(\underline{\mathrm{CH}}_{3}\right)_{3}\right), 45.0\left(\mathrm{CH}_{2} \mathrm{~N}\right), 48.7(\mathrm{CHN}), 79.7\left(\underline{\mathrm{C}}\left(\mathrm{CH}_{3}\right)_{3}\right), 79.8\left(\underline{\mathrm{C}}\left(\mathrm{CH}_{3}\right)_{3}\right), 112.9$ (CH indol), $113.0(\mathrm{C}$ indol), $121.6(\mathrm{CH}$ indol), 122.1 (C indol), $122.9(\mathrm{CH}$ indol), $125.2(\mathrm{CH}$ indol), 127.5 (C indol), $135.2(\mathrm{C}$ indol), $156.0(\mathrm{C}=\mathrm{O}), 156.6(\mathrm{C}=\mathrm{O})$. LRMS (ESI): $\mathrm{m} / \mathrm{z}=476$ and $478\left[(\mathrm{M}+\mathrm{Na})^{+}\right]$. Anal. Calcd for $\mathbf{C}_{20} \mathbf{H}_{28} \mathbf{N}_{3} \mathbf{O}_{4} \mathbf{B r}: \mathrm{C}, 52.87 ; \mathrm{H}, 6.22 ; \mathrm{N}, 9.25$. Found: $\mathrm{C}$, 52.94; H, 6.43; N, 9.01.

\section{- 1-[N-(tert-butoxycarbonyl)amino]-2-[ $N$-(tert-butoxycarbonyl)amino]-1-(6'-} bromoindol-3'-yl)ethane 12c

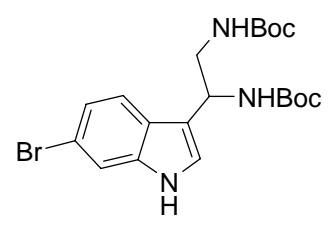

$N$-Hydroxylamine $11 \mathrm{f}(85 \mathrm{mg}, 0.18 \mathrm{mmol})$ was dissolved into $3 \mathrm{~mL}$ of THF under inert atmosphere. 16 equiv of ungazed water $(0.05 \mathrm{~mL}, 2.9 \mathrm{mmol})$ were added, immediately followed by 4 equiv of a $\mathrm{SmI}_{2}$ solution in THF $(0.72 \mathrm{mmol}, 7.2 \mathrm{~mL})$. The reaction was allowed to stir at room temperature during one hour. It was then quenched by air, and then by the addition of a saturated aqueous solution of sodium thiosulfate. The resulting aqueous mixture was extracted three times by EtOAc. Combined organic layers were washed with brine and dried over anhydrous magnesium sulfate. After the removal of the solvent, the residue was purified by column chromatography (Eluent: $\mathrm{Et}_{2} \mathrm{O}$ /pentane, 1:1). The amine 12c was obtained as a colorless solid (43 $\mathrm{mg}, 0.095 \mathrm{mmol})$.

Yield: 53\%. IR (film): 3316, 2981, 2931, 1701, 1520, 1459, 1393, 1366, 1256, 1168, 1025, $800 \mathrm{~cm}^{-1} .{ }^{1} \mathbf{H}$ NMR $\left(\mathbf{C D}_{3} \mathbf{C O C D}_{3}, 300 \mathbf{M H z}\right): \delta=1.41\left(\mathrm{~s}, 18 \mathrm{H}, \mathrm{C}\left(\underline{\mathrm{CH}}_{3}\right)_{3}\right), 3.45-3.65(\mathrm{~m}, 2 \mathrm{H}$, $\mathrm{CH}_{2} \mathrm{~N}$ ), 5.15 (dd, $J=8.2$ and $14.2 \mathrm{~Hz}, 1 \mathrm{H}, \mathrm{CHN}$ ), 6.09 (br s, $1 \mathrm{H}, \mathrm{NHBoc}$ ), 6.10 (br s, $1 \mathrm{H}$, NHBoc), 7.16 (dd, $J=1.8$ and $8.4 \mathrm{~Hz}, 1 \mathrm{H}, \mathrm{H}$ indol), 7.35 (d, $J=2.4 \mathrm{~Hz}, 1 \mathrm{H}, \mathrm{H}$ indol), 7.59 (d, 
$J=1.7 \mathrm{~Hz}, 1 \mathrm{H}, \mathrm{H}$ indol), 7.66 (d, $J=6.5 \mathrm{~Hz}, 1 \mathrm{H}, \mathrm{H}$ indol), 10.28 (s, $1 \mathrm{H}, \mathrm{NH}$ indol). ${ }^{13} \mathbf{C}$ NMR $\left(\mathrm{CD}_{3} \mathbf{C O C D}_{3}, 75.5 \mathrm{MHz}\right): \delta=28.6\left(2 \mathrm{C}\left(\underline{\mathrm{CH}}_{3}\right)_{3}\right), 45.6\left(\mathrm{CH}_{2} \mathrm{~N}\right), 48.9(\mathrm{CHN}), 78.8\left(2 \underline{\mathrm{C}}\left(\mathrm{CH}_{3}\right)_{3}\right)$, 115.0 (CH indol), 115.4 (C indol), $116.7(\mathrm{C}$ indol), 121.4 ( $\mathrm{CH}$ indol), $122.7(\mathrm{CH}$ indol), 123.9 (CH indol), 126.4 (C indol), 138.5 (C indol), $156.4(\mathrm{C}=\mathrm{O}), 157.1$ ( $\mathrm{C}=\mathrm{O})$. LRMS (ESI): $\mathrm{m} / \mathrm{z}=$ 476 and $478\left[(\mathrm{M}+\mathrm{Na})^{+}\right], 492$ and $494\left[(\mathrm{M}+\mathrm{K})^{+}\right]$. 


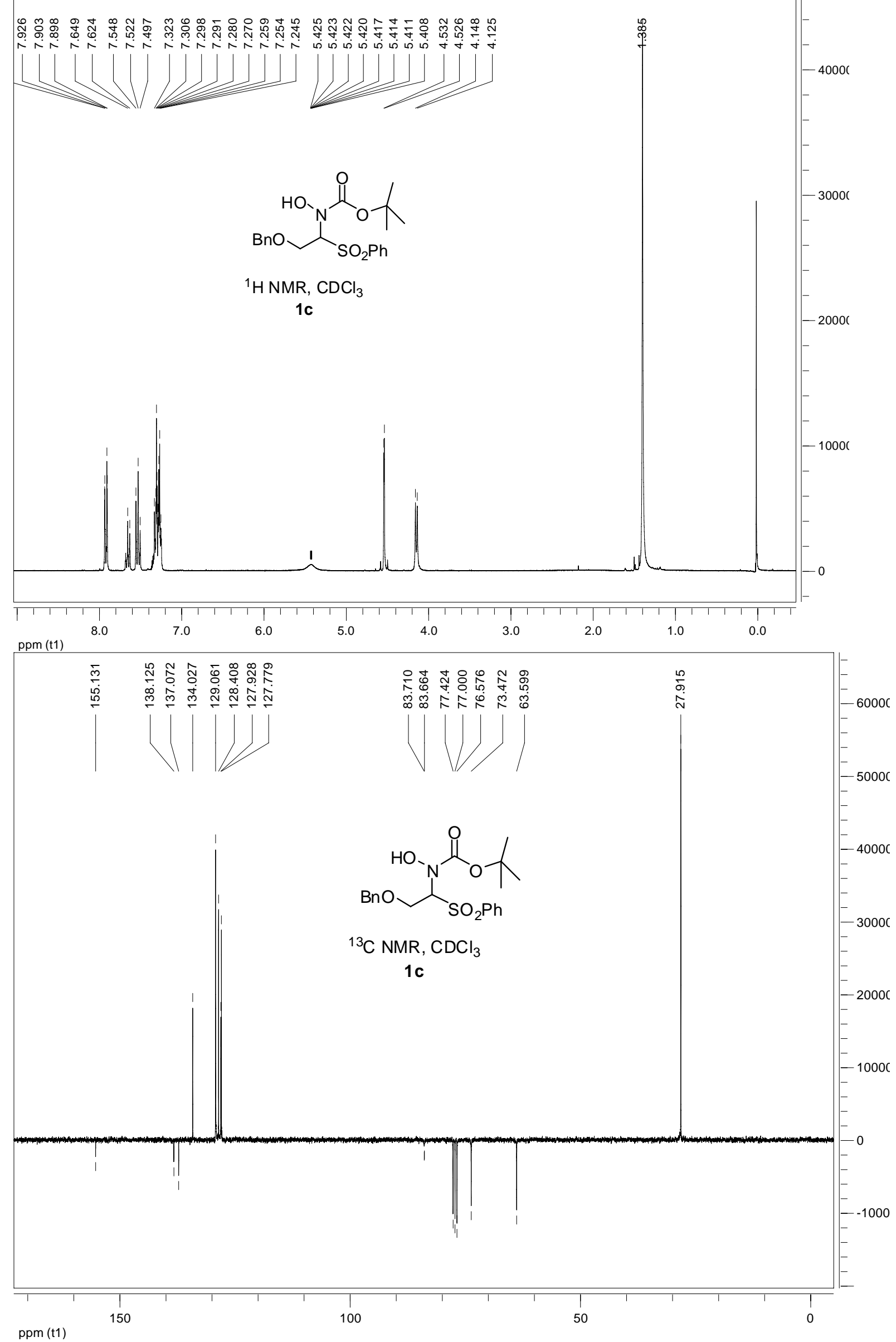




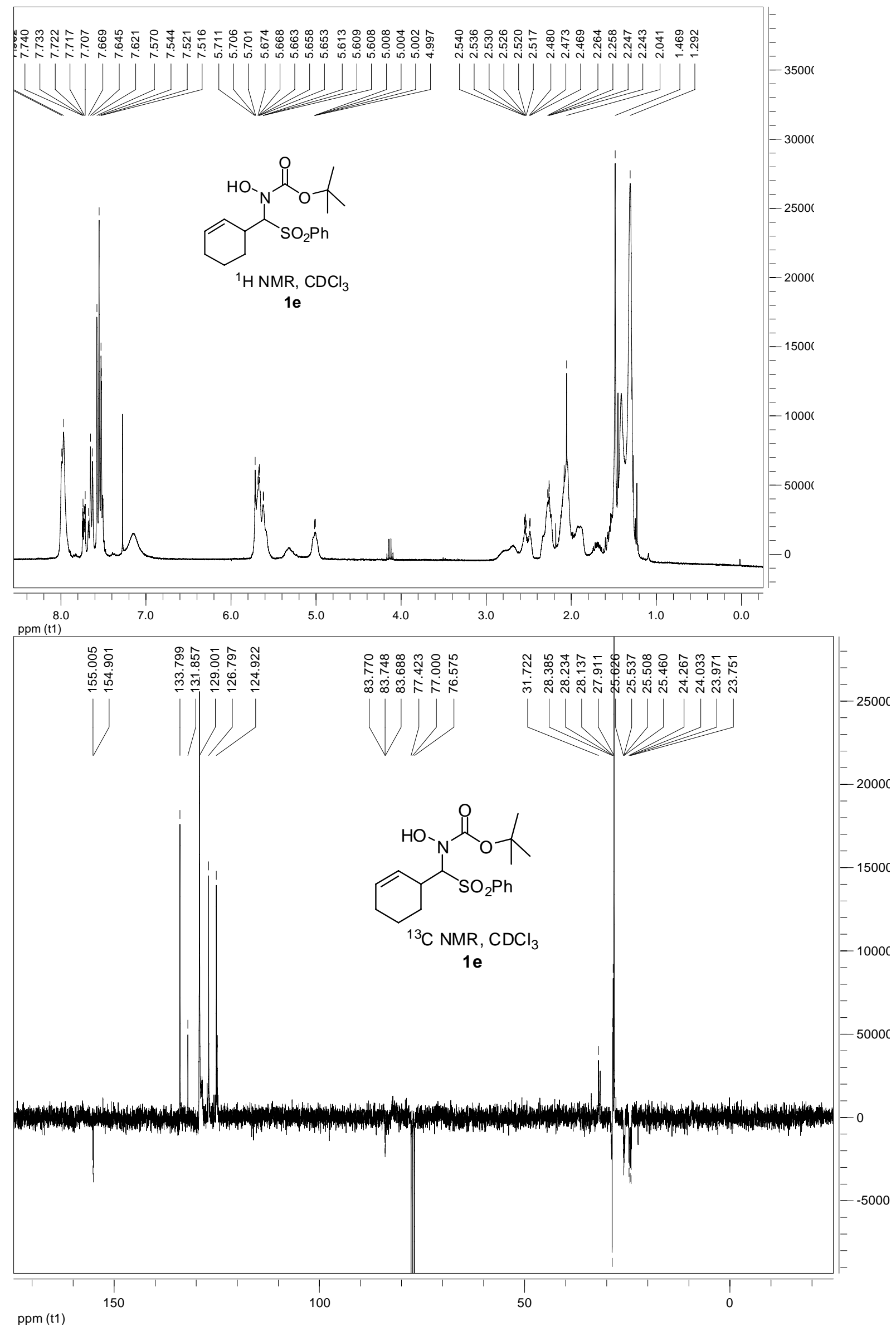




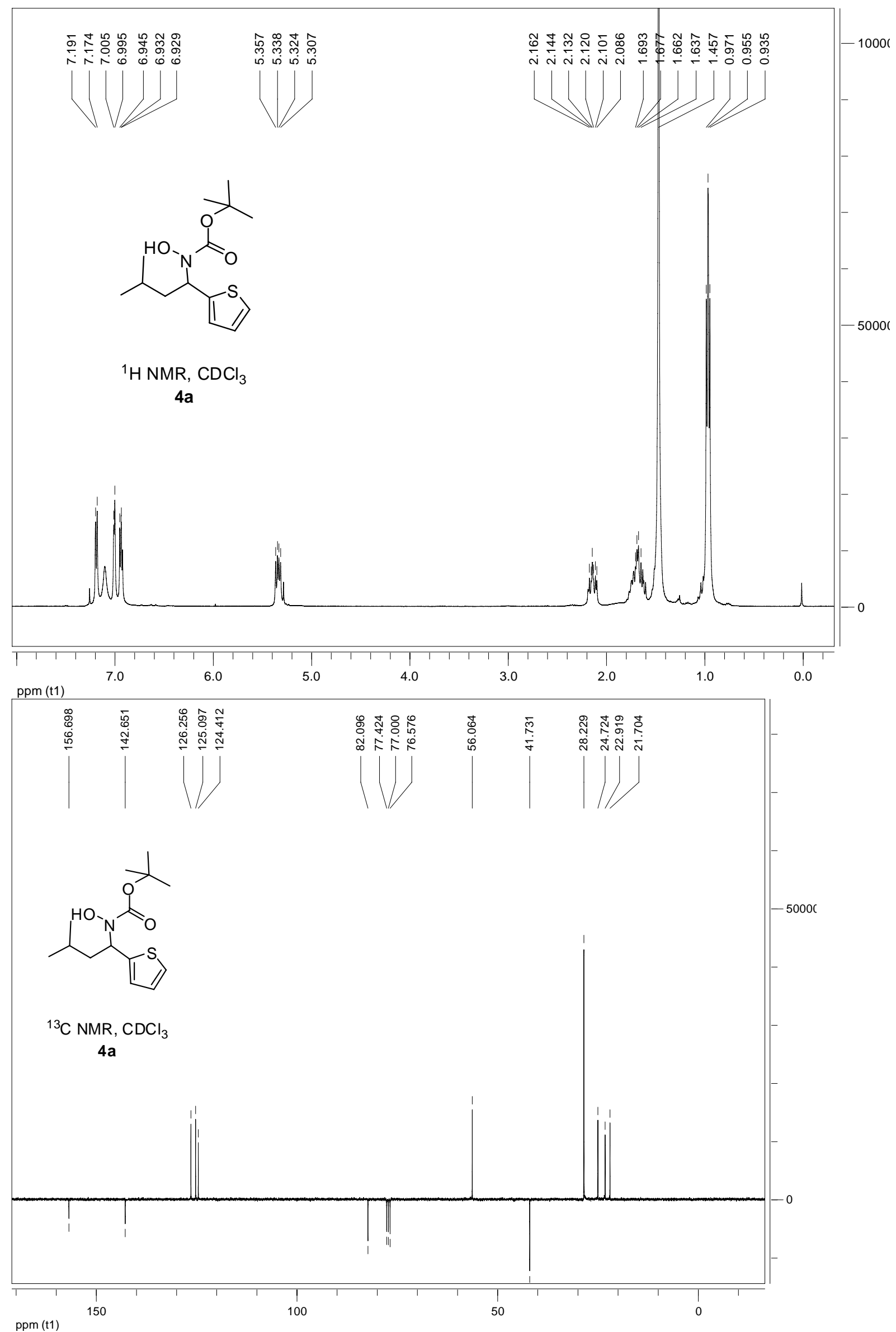




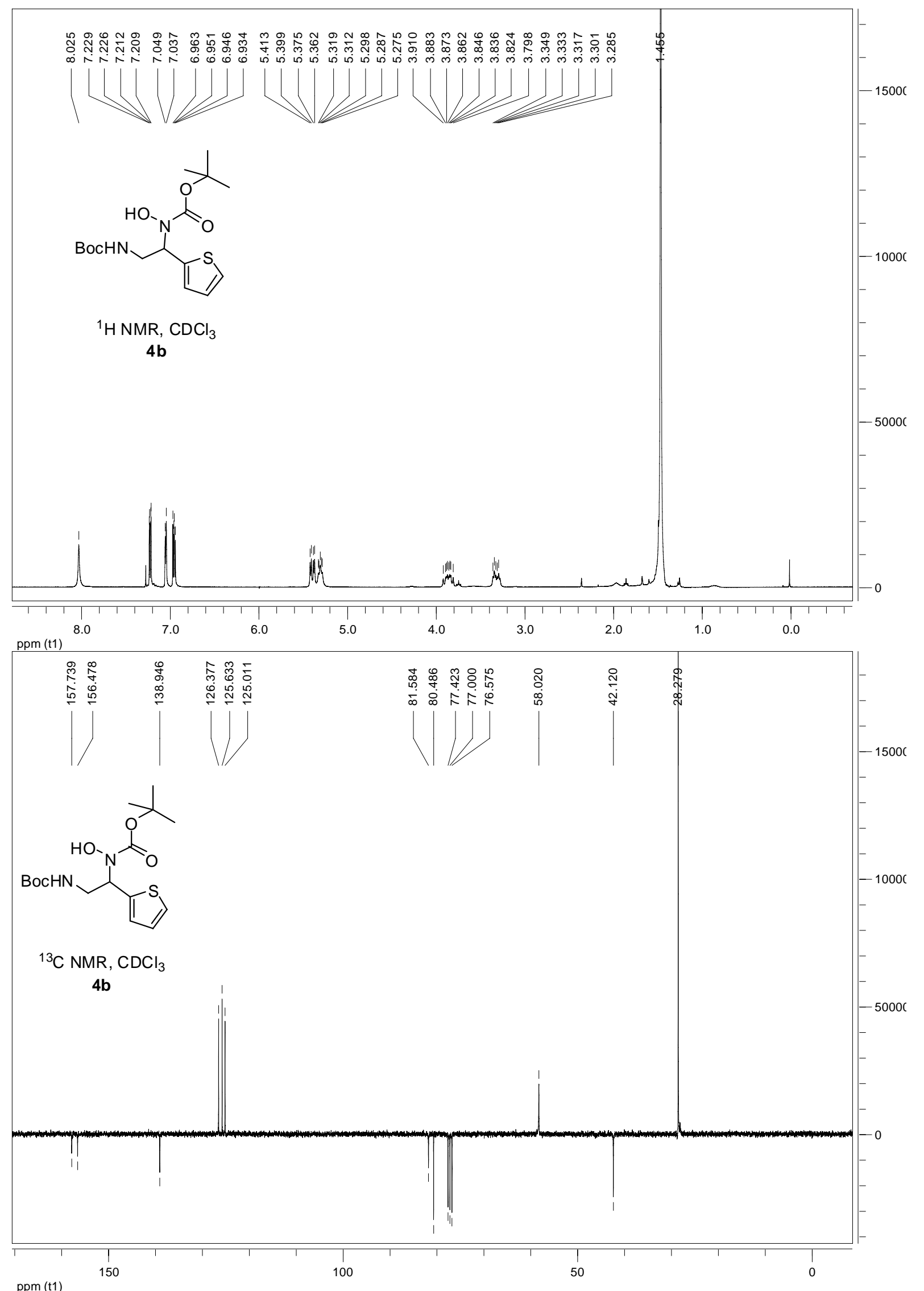




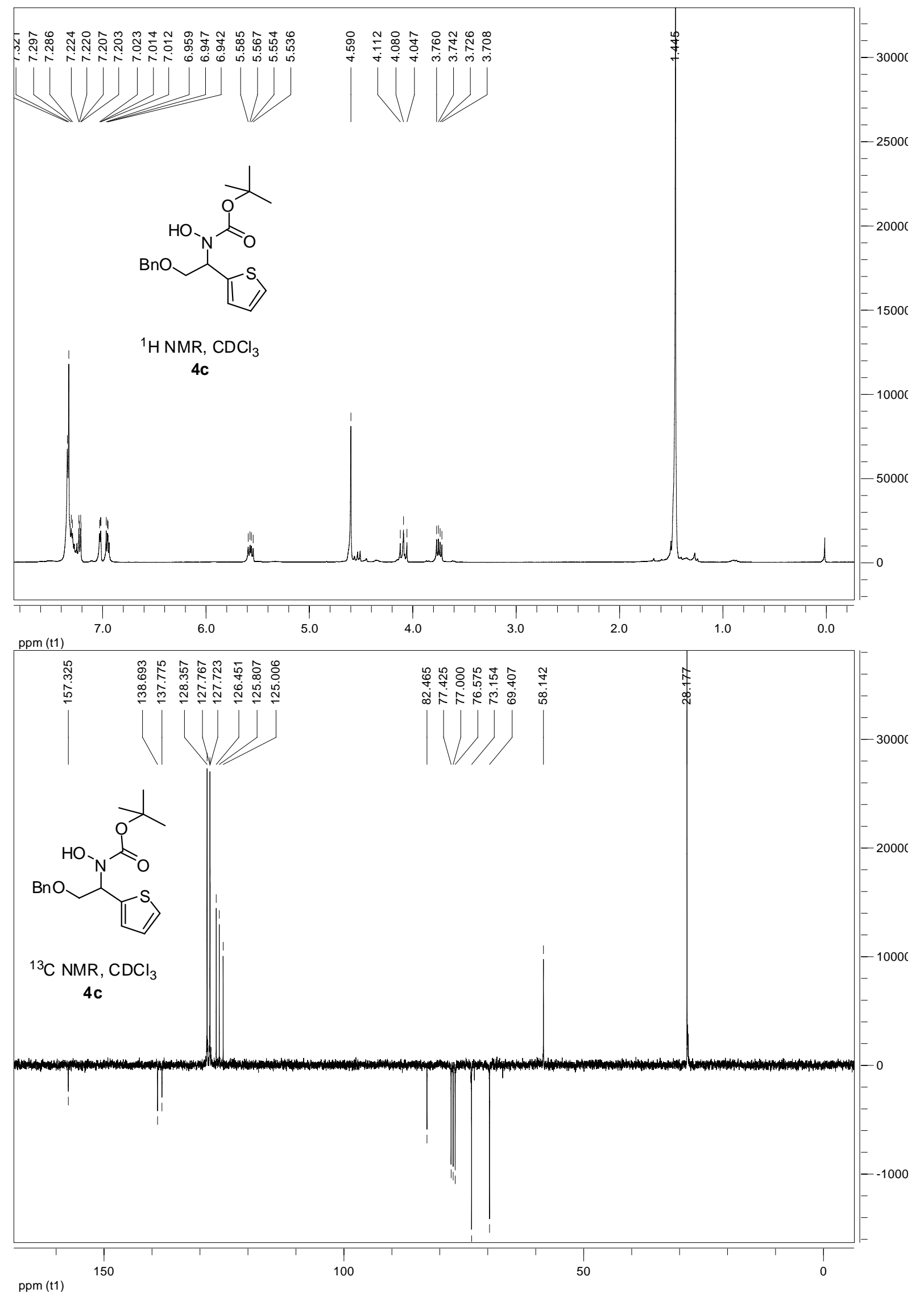




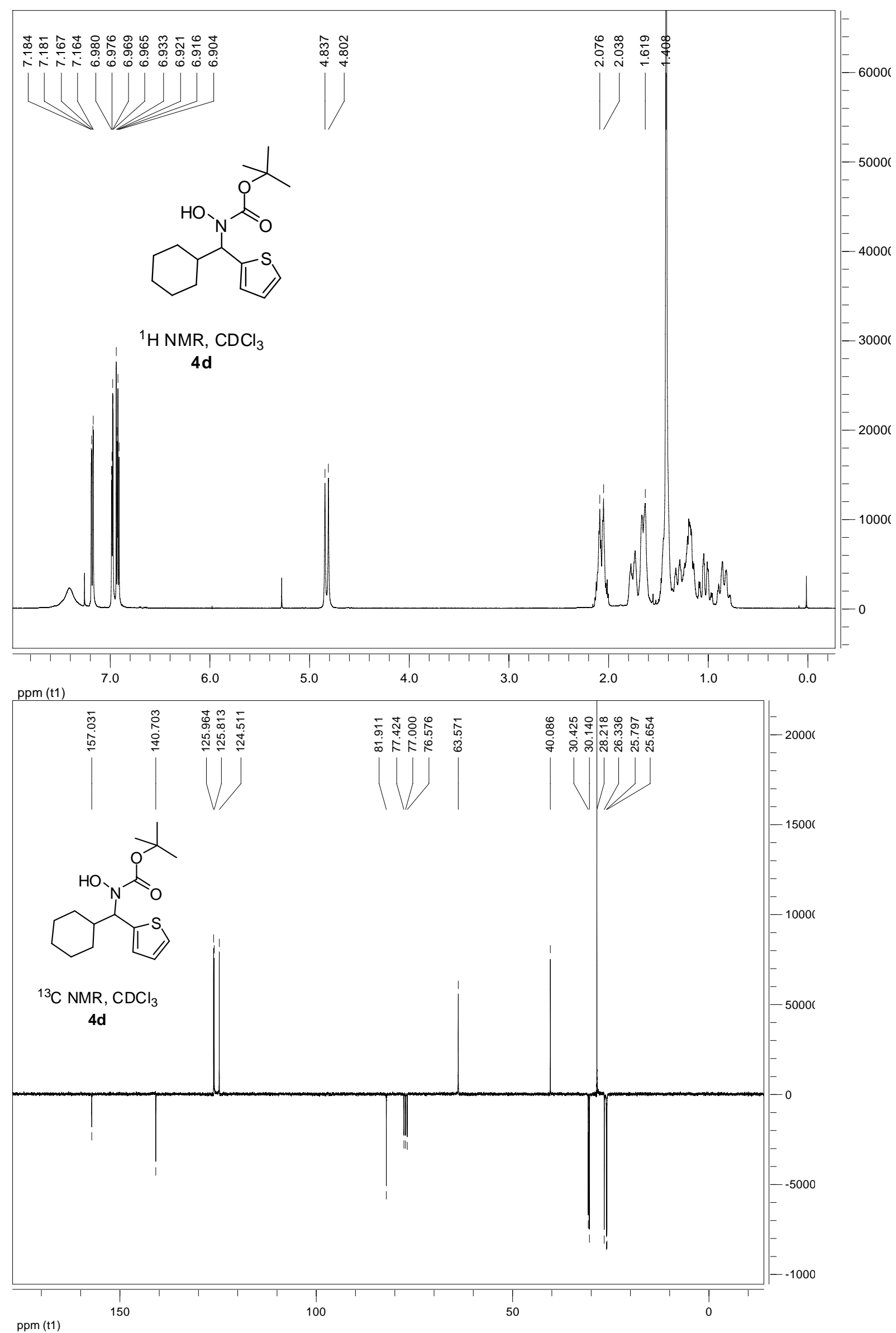



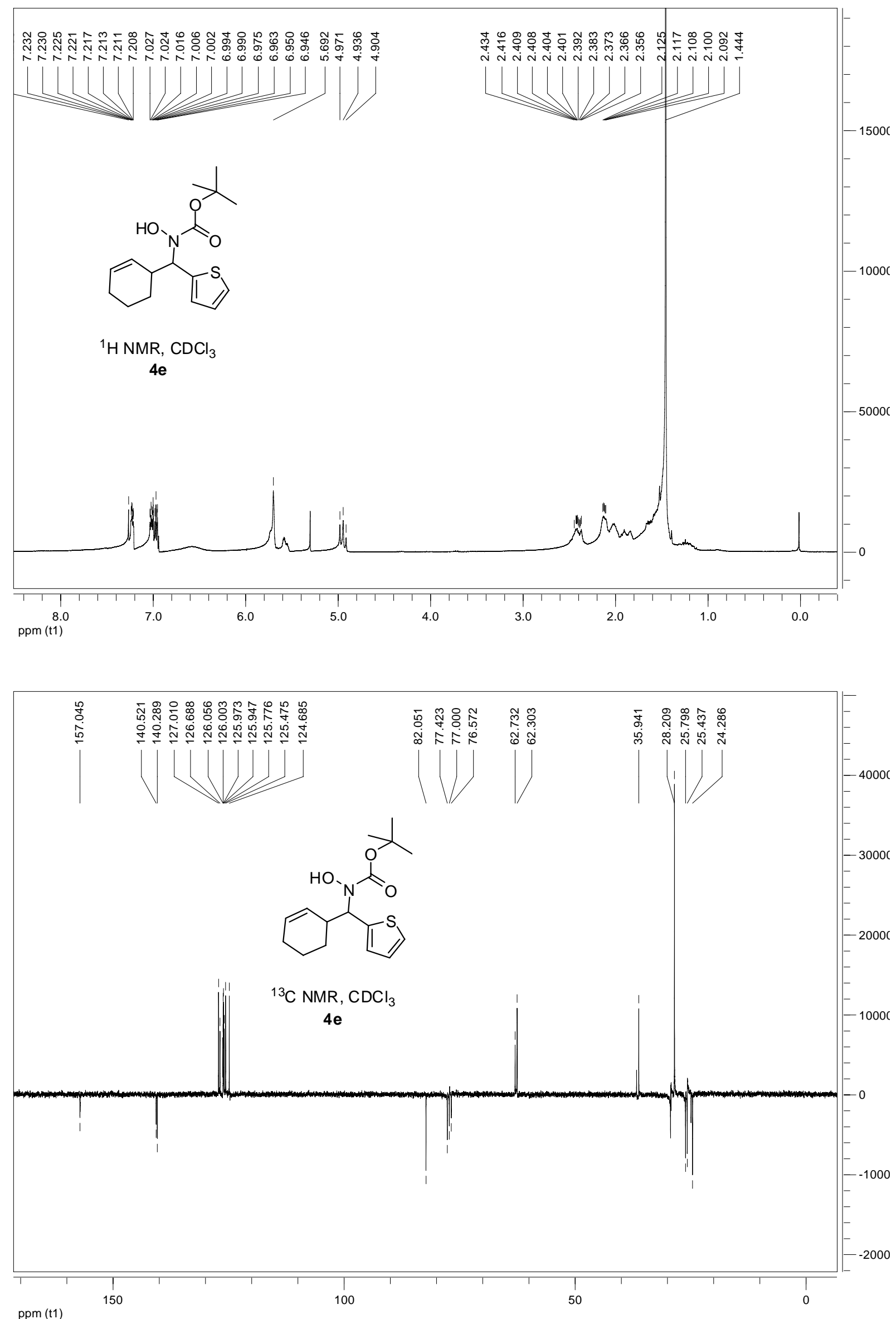


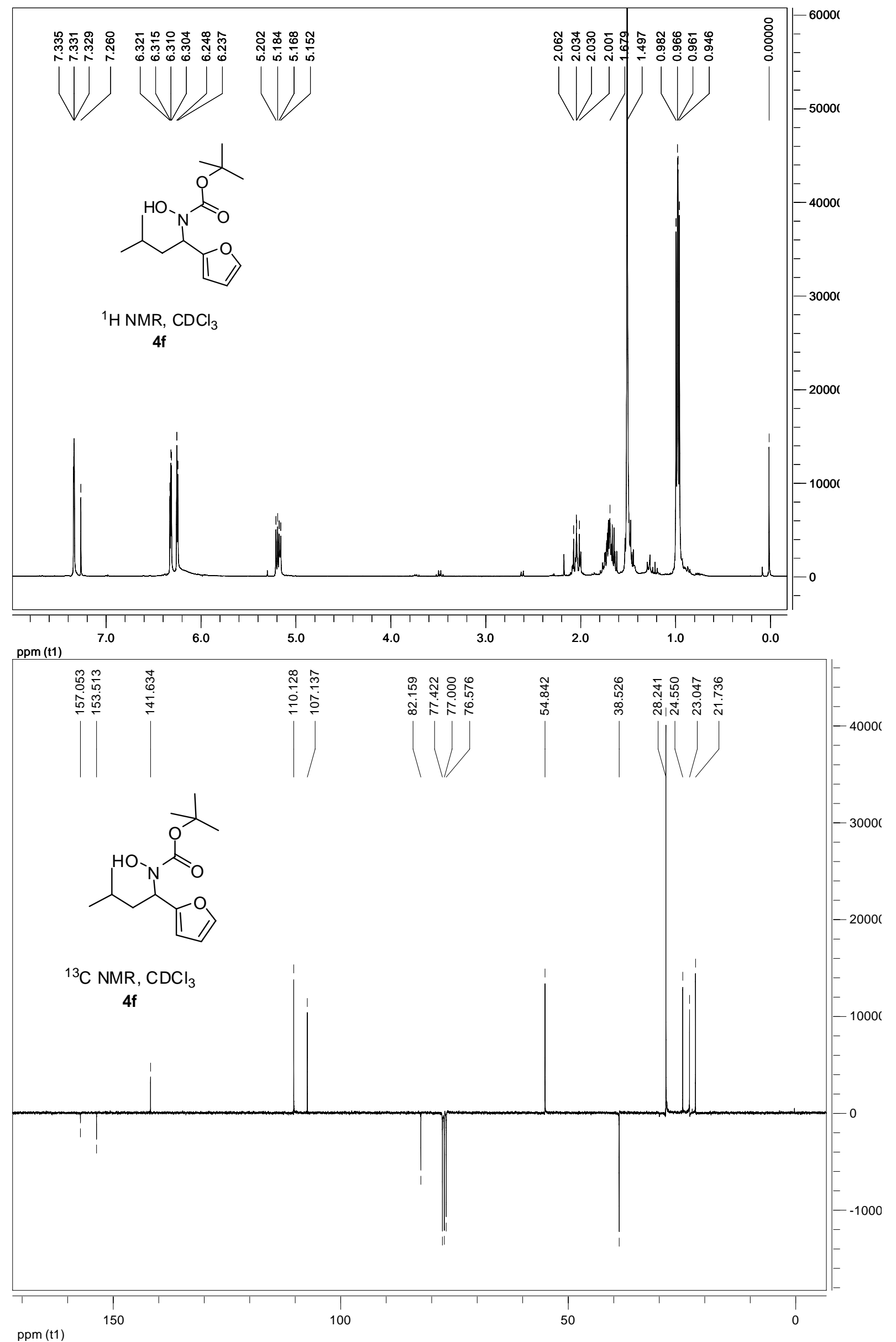




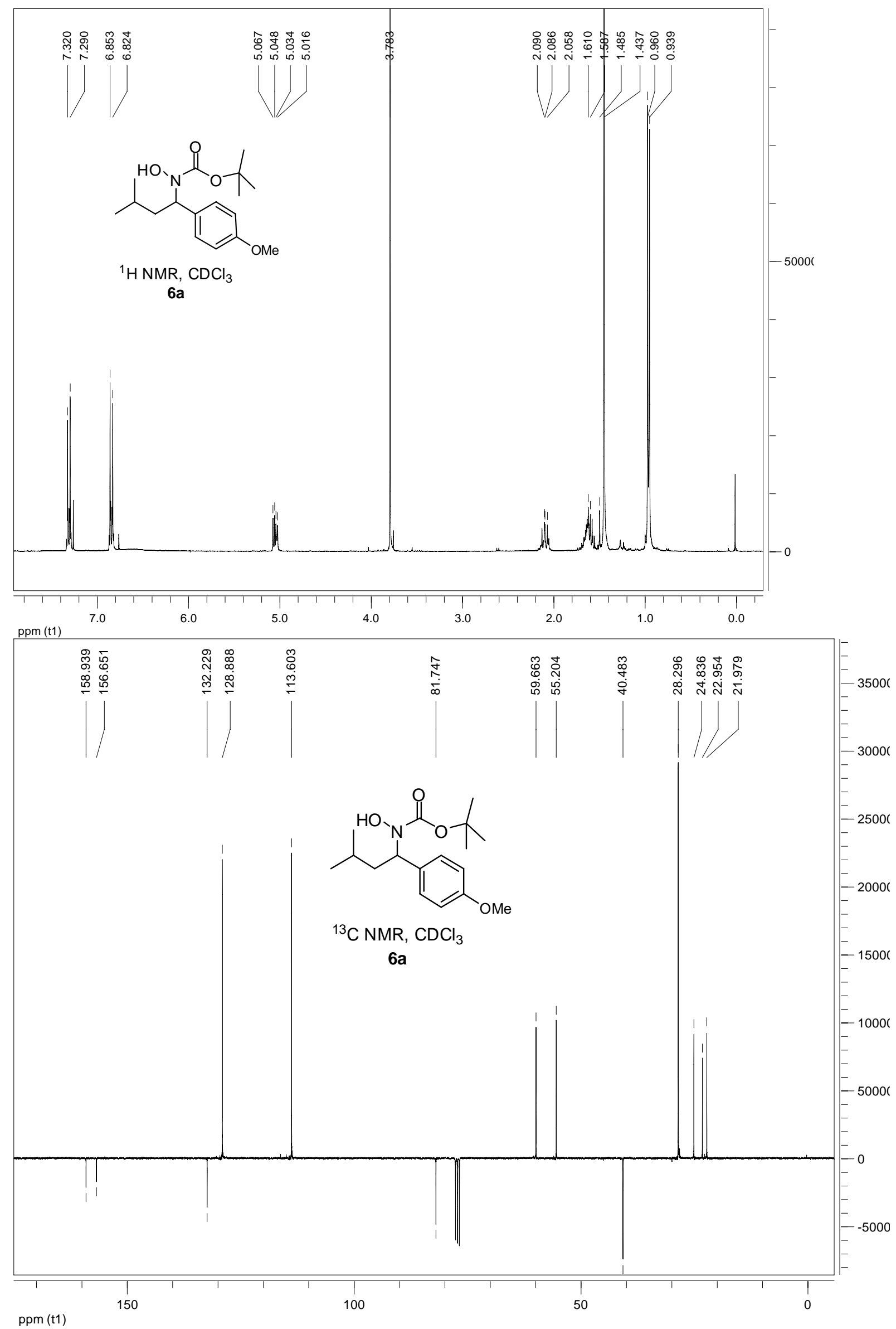




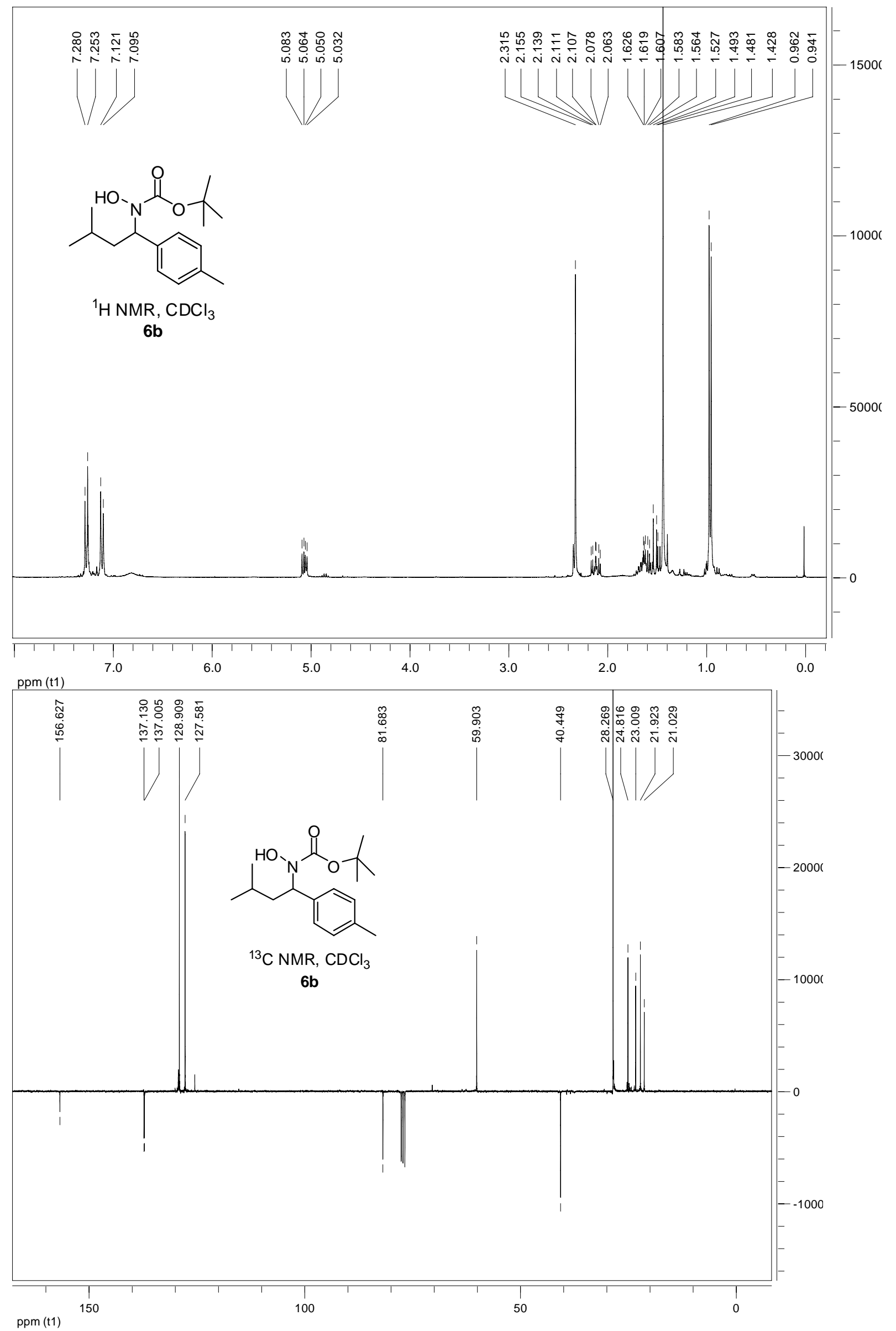




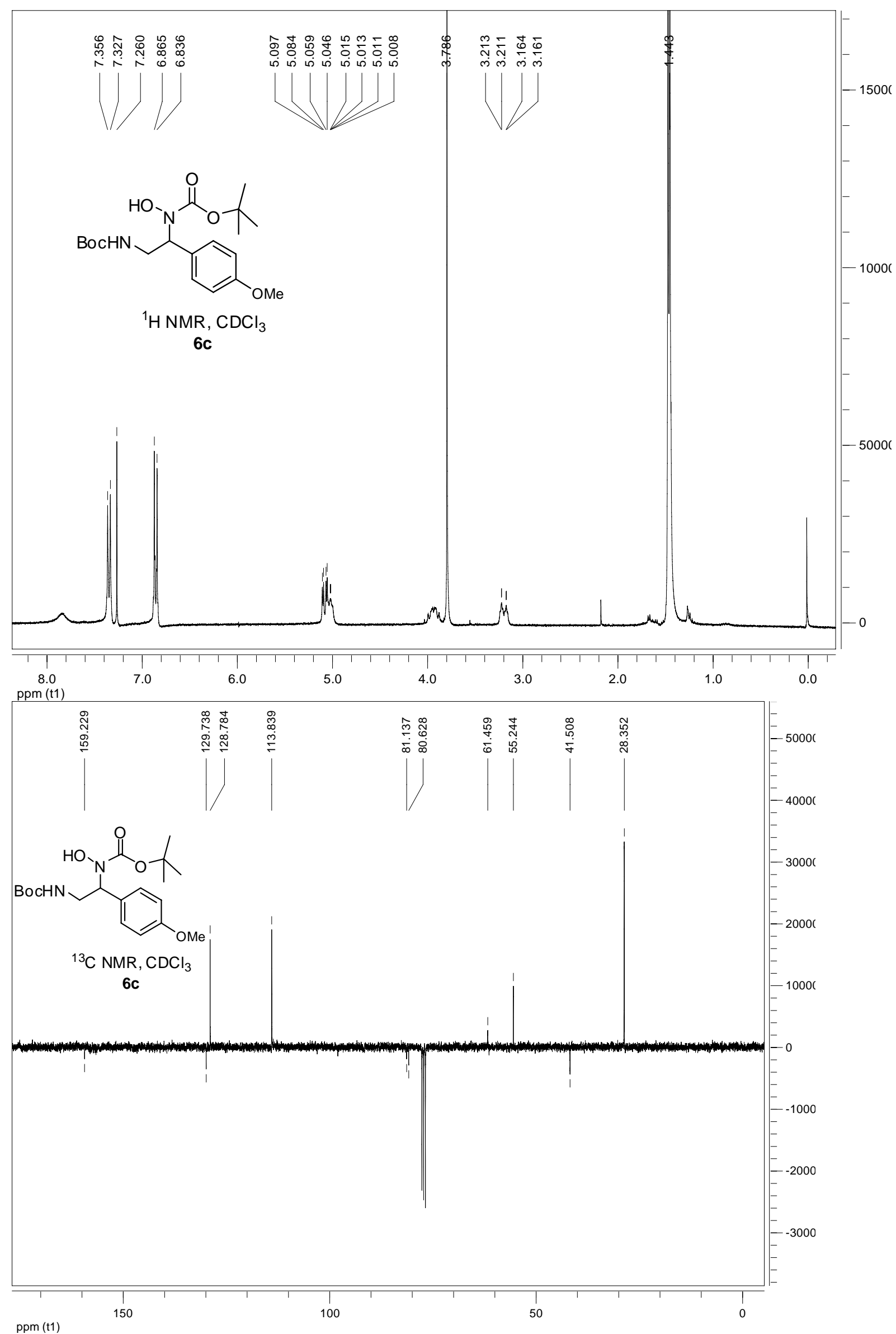




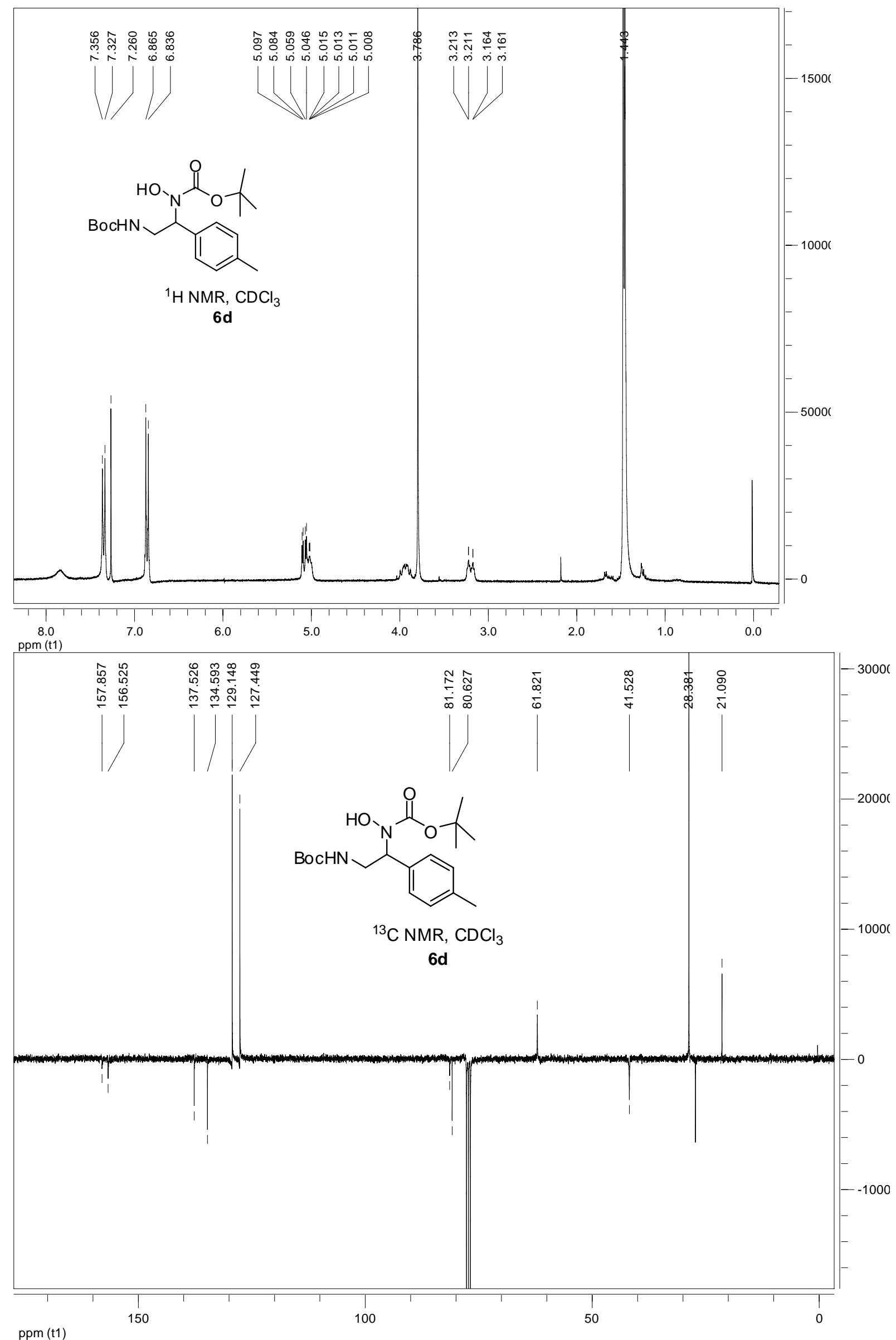




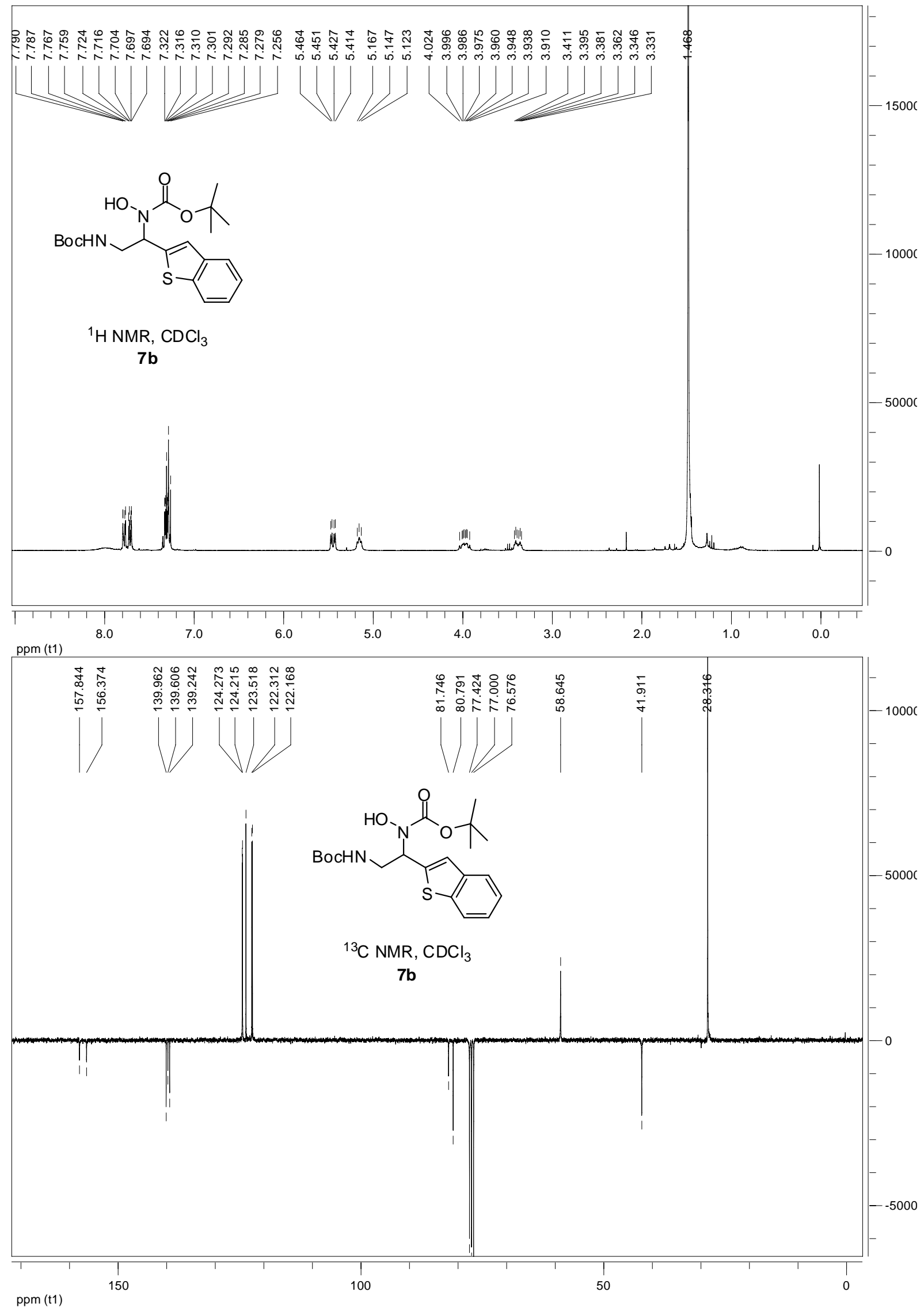




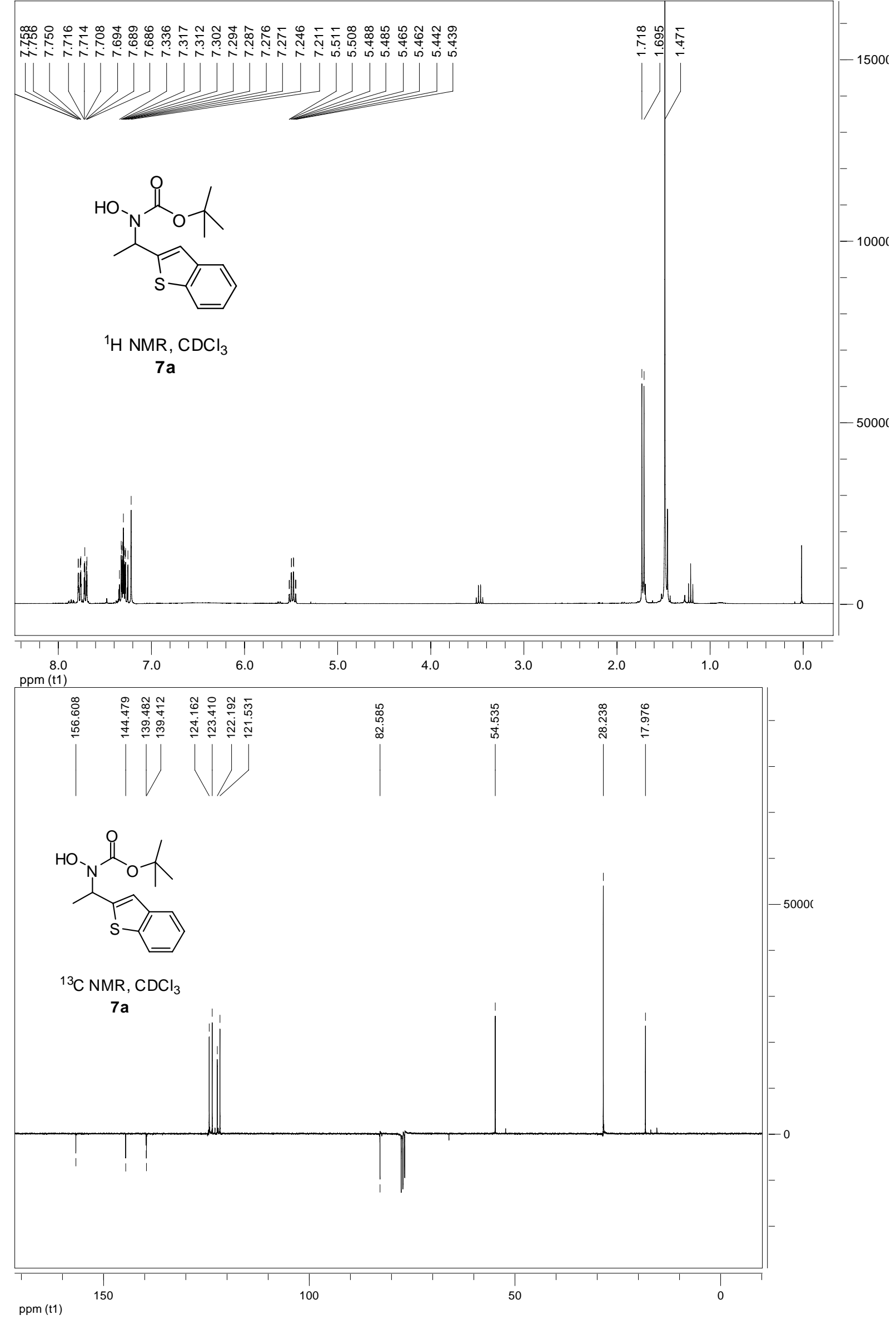




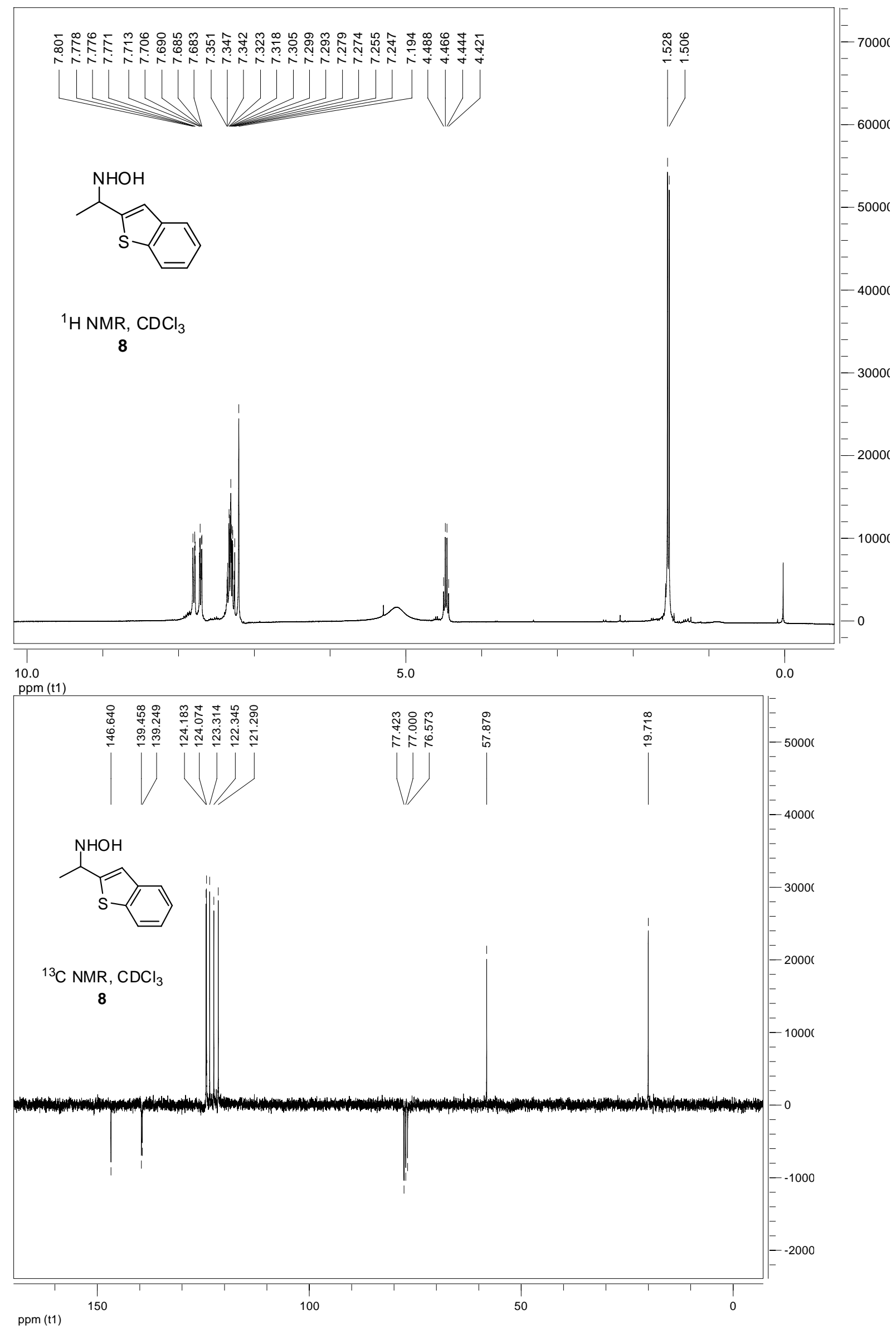




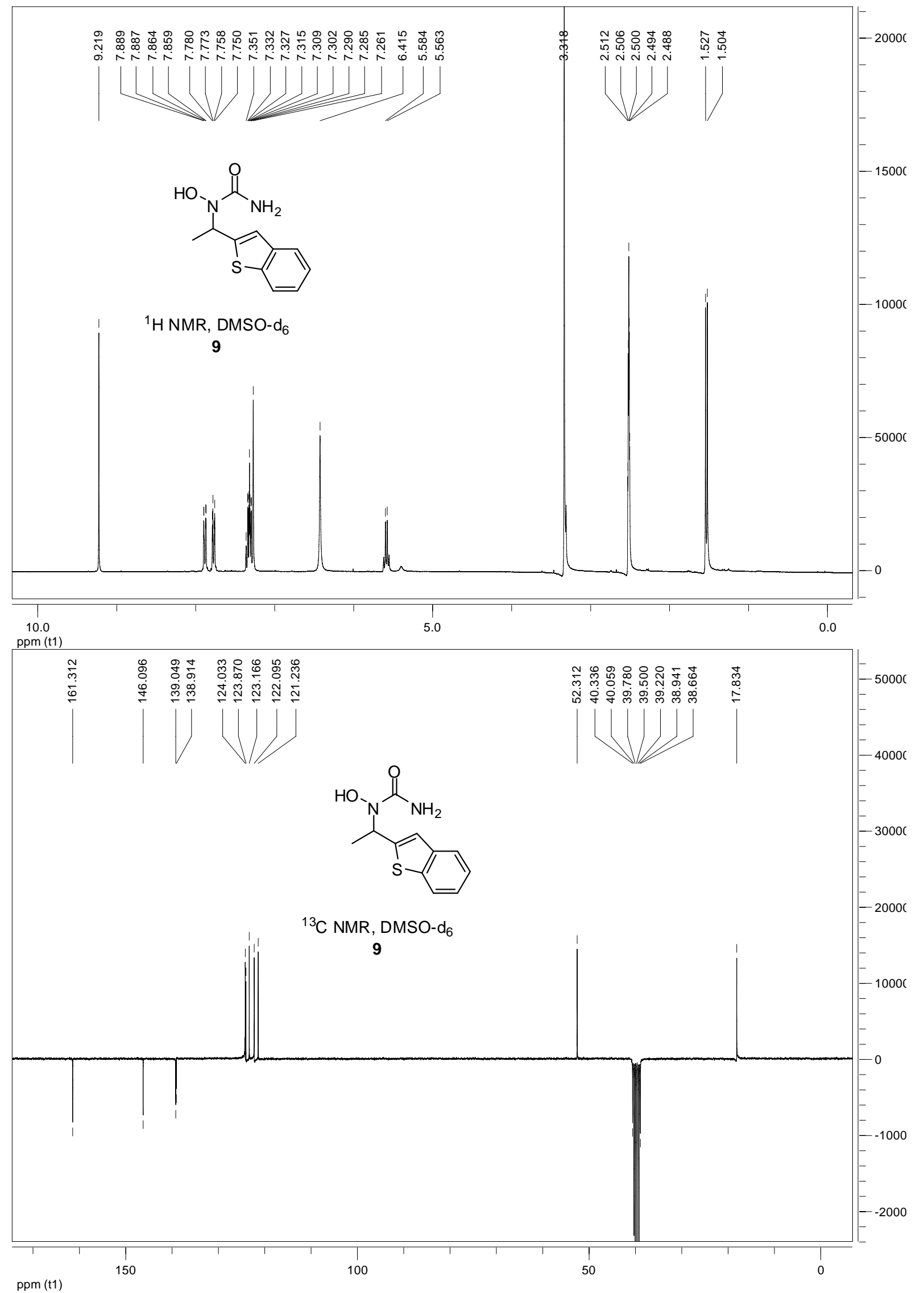




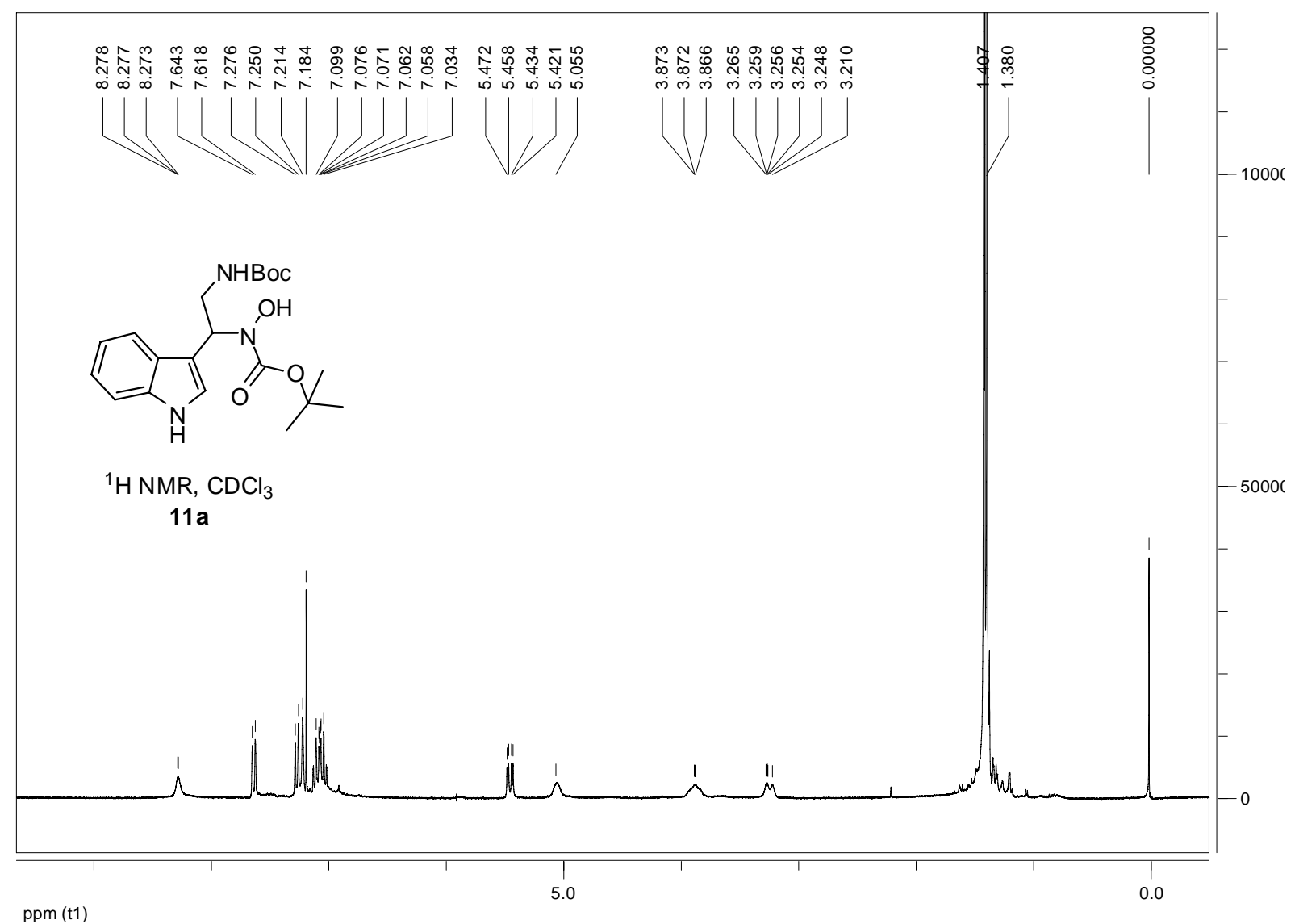




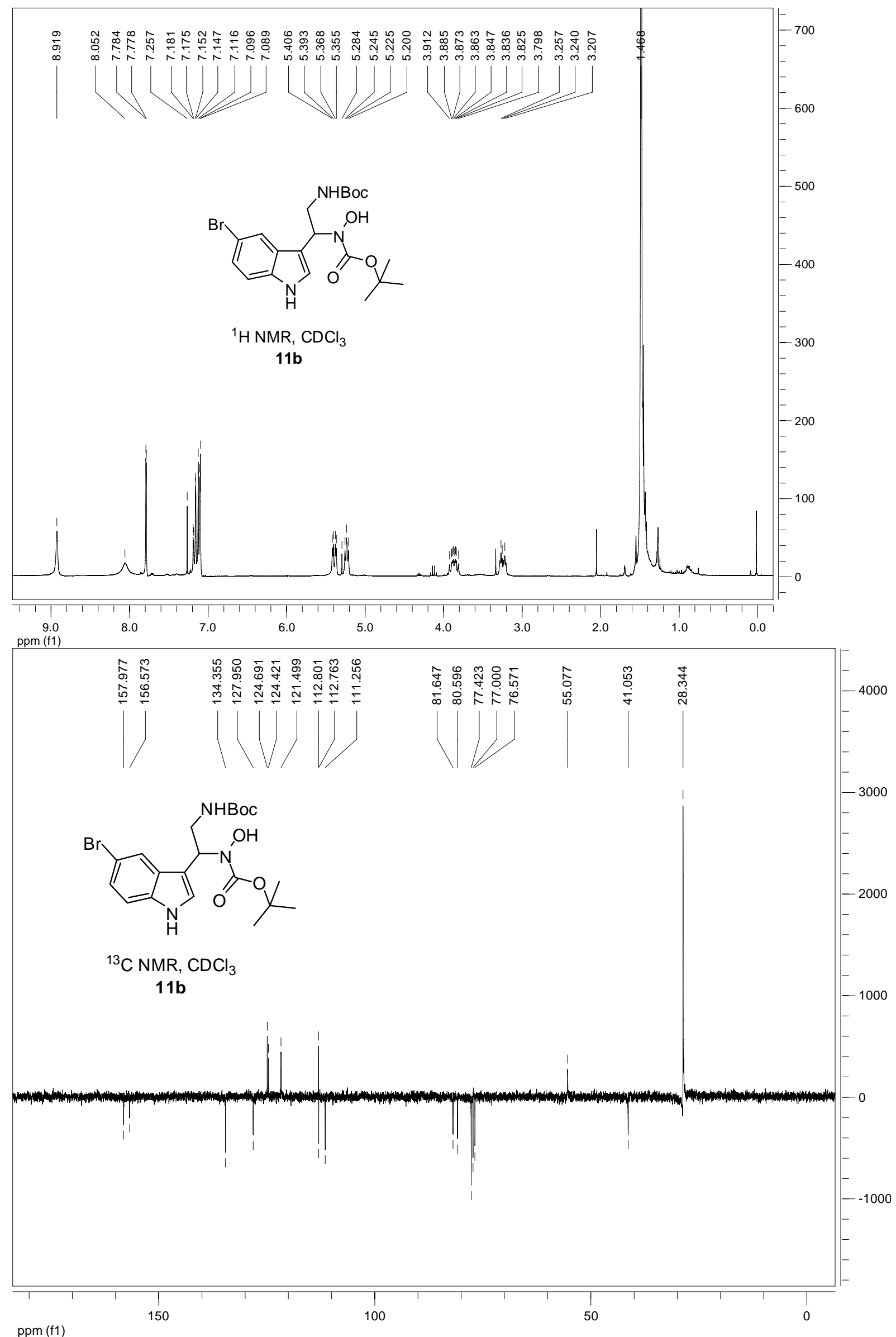




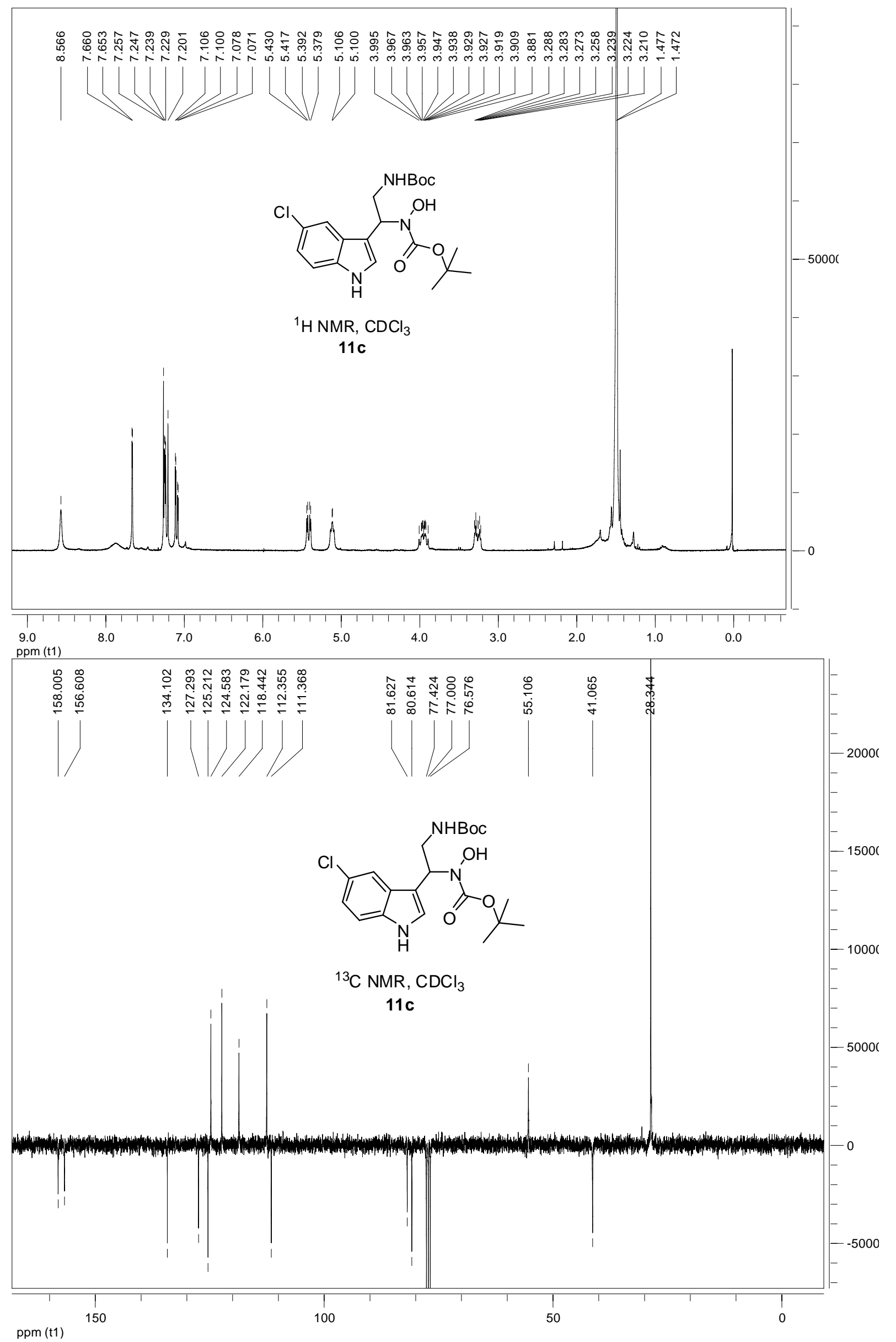




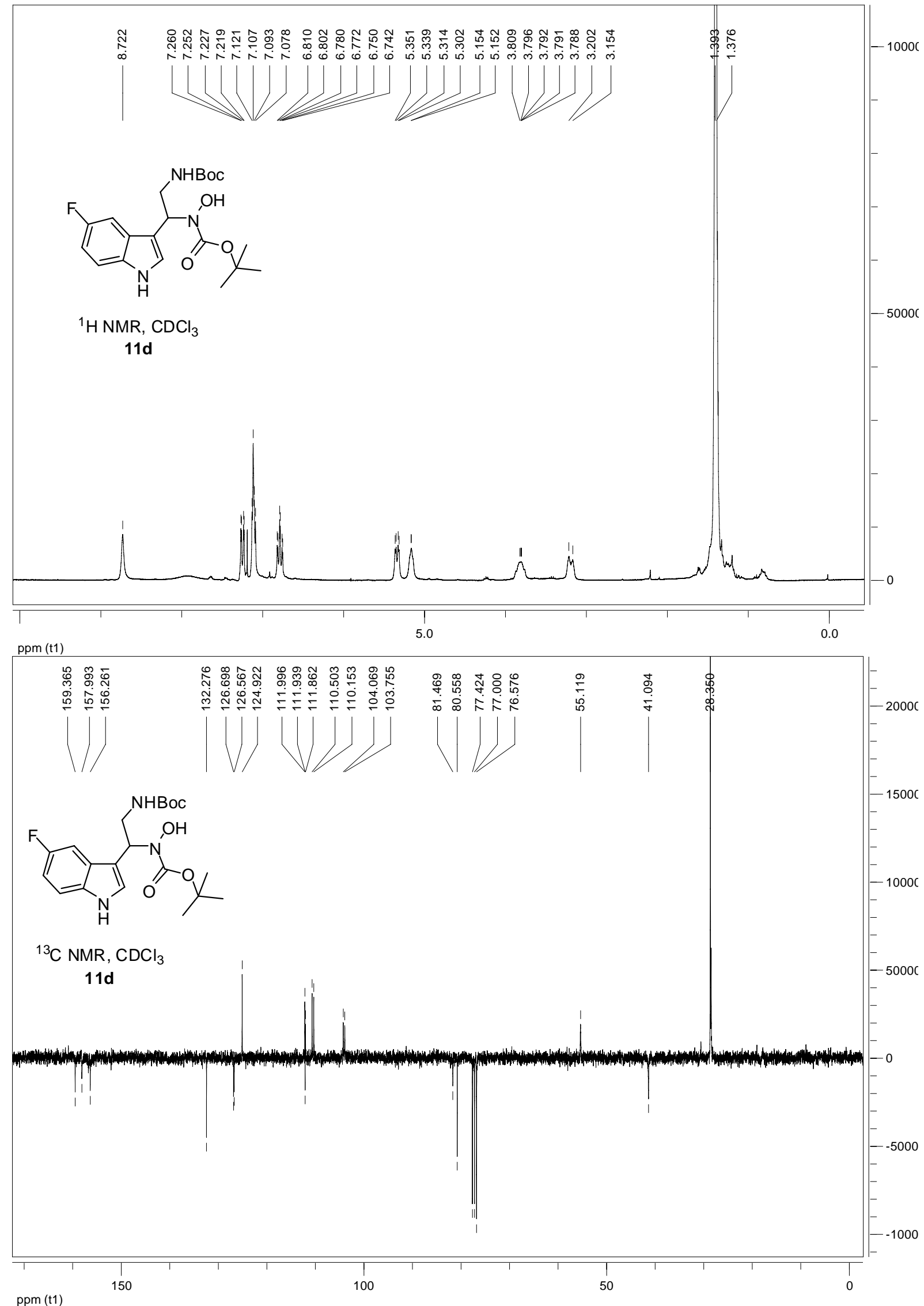




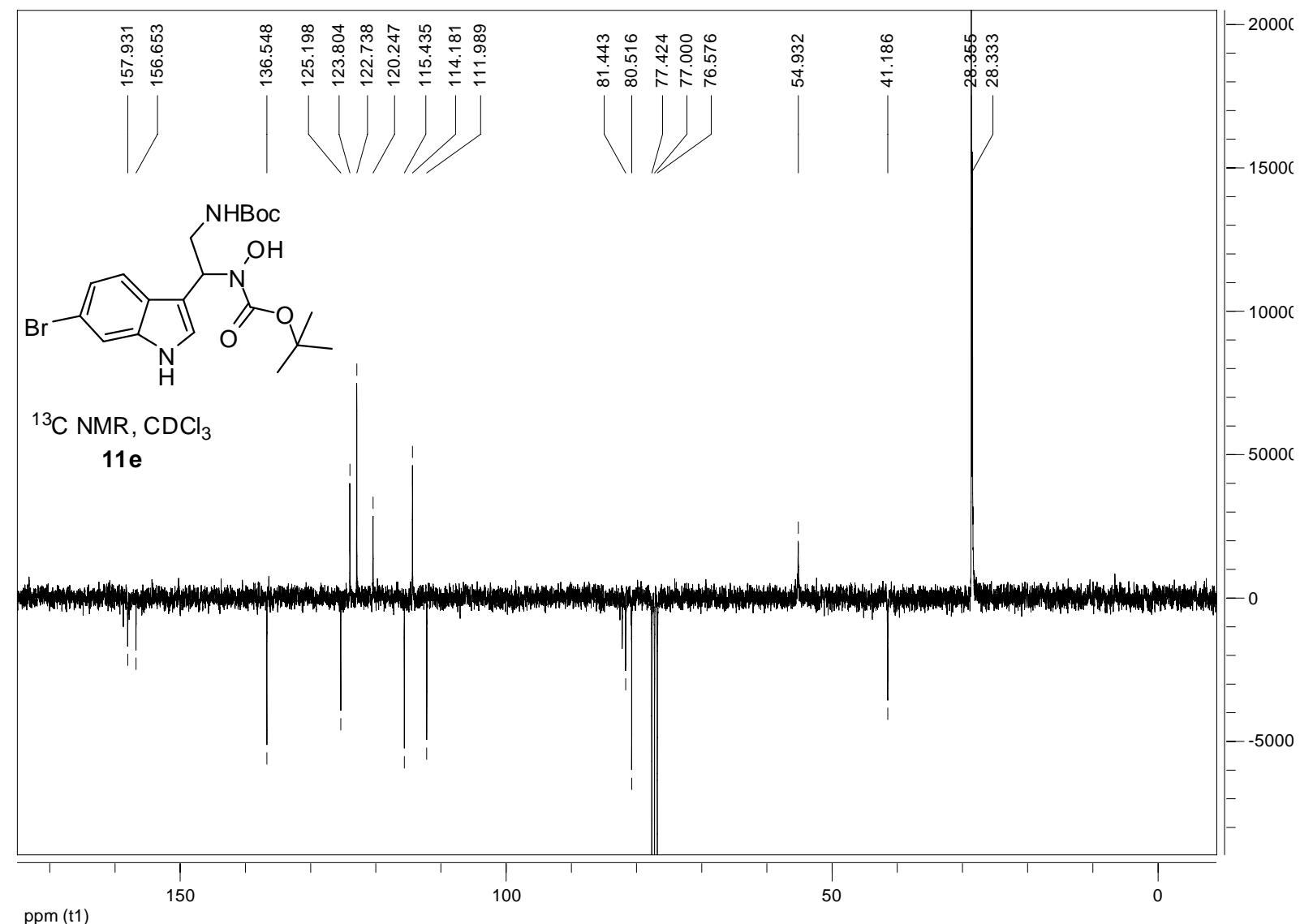




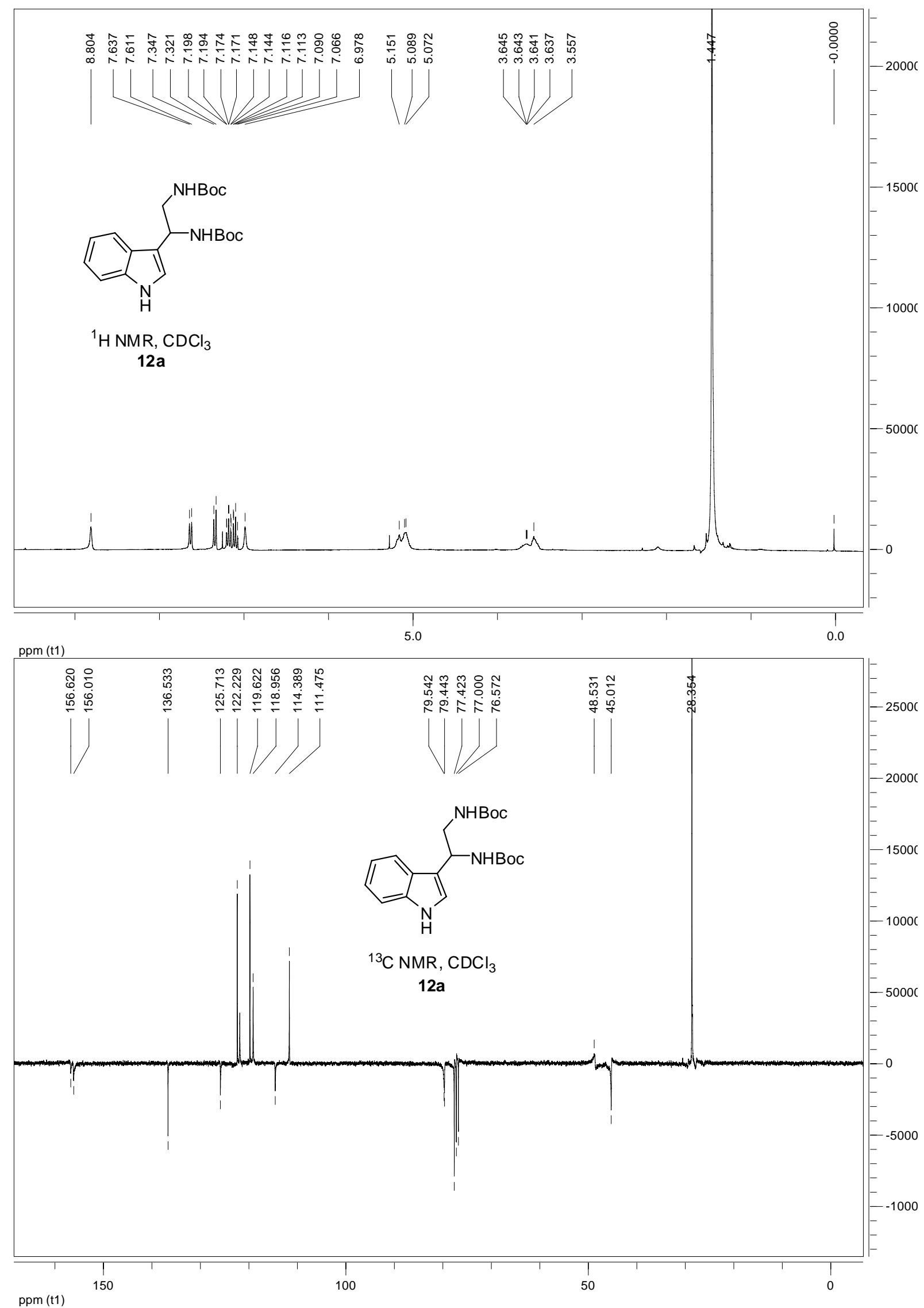




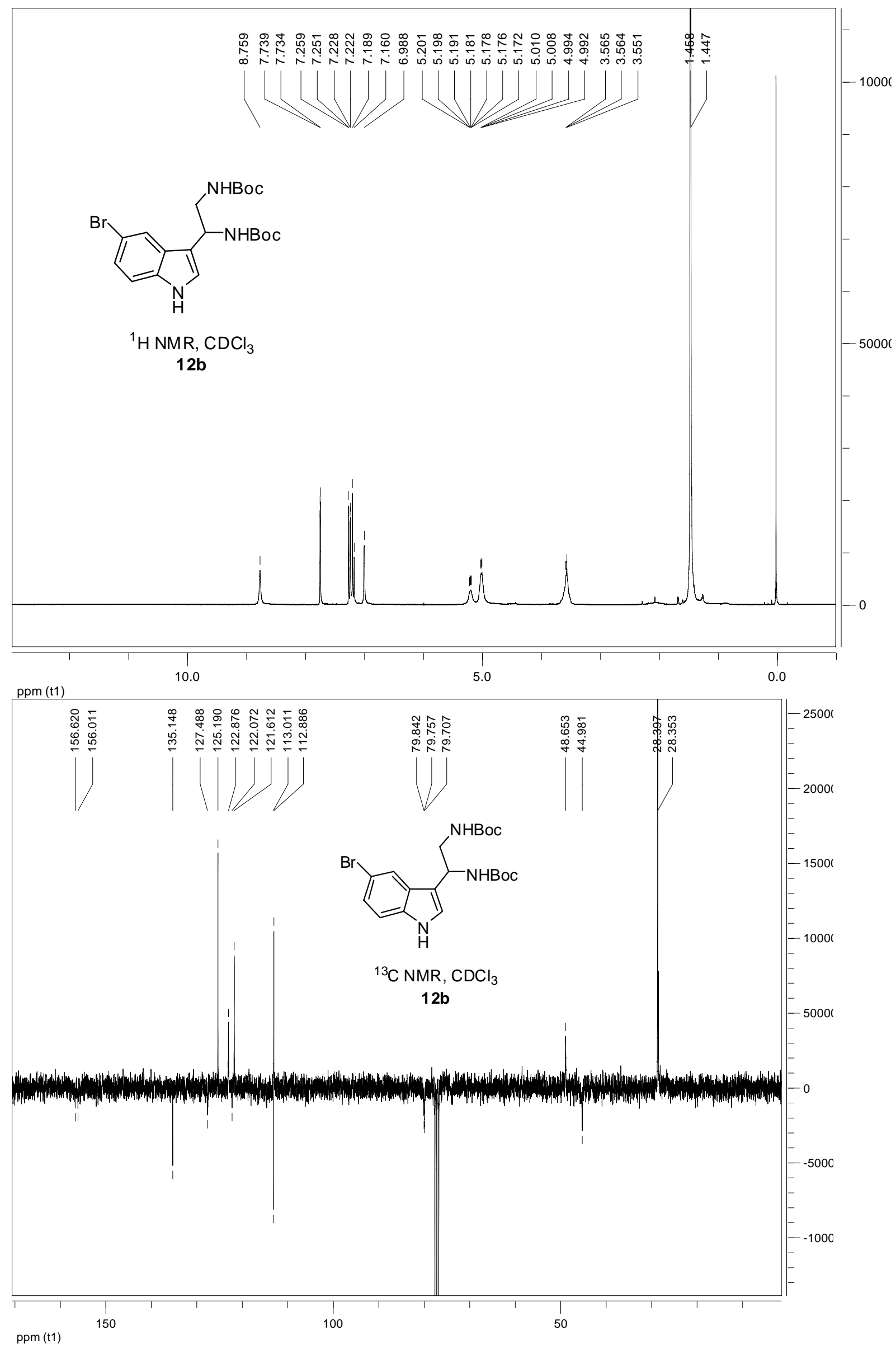



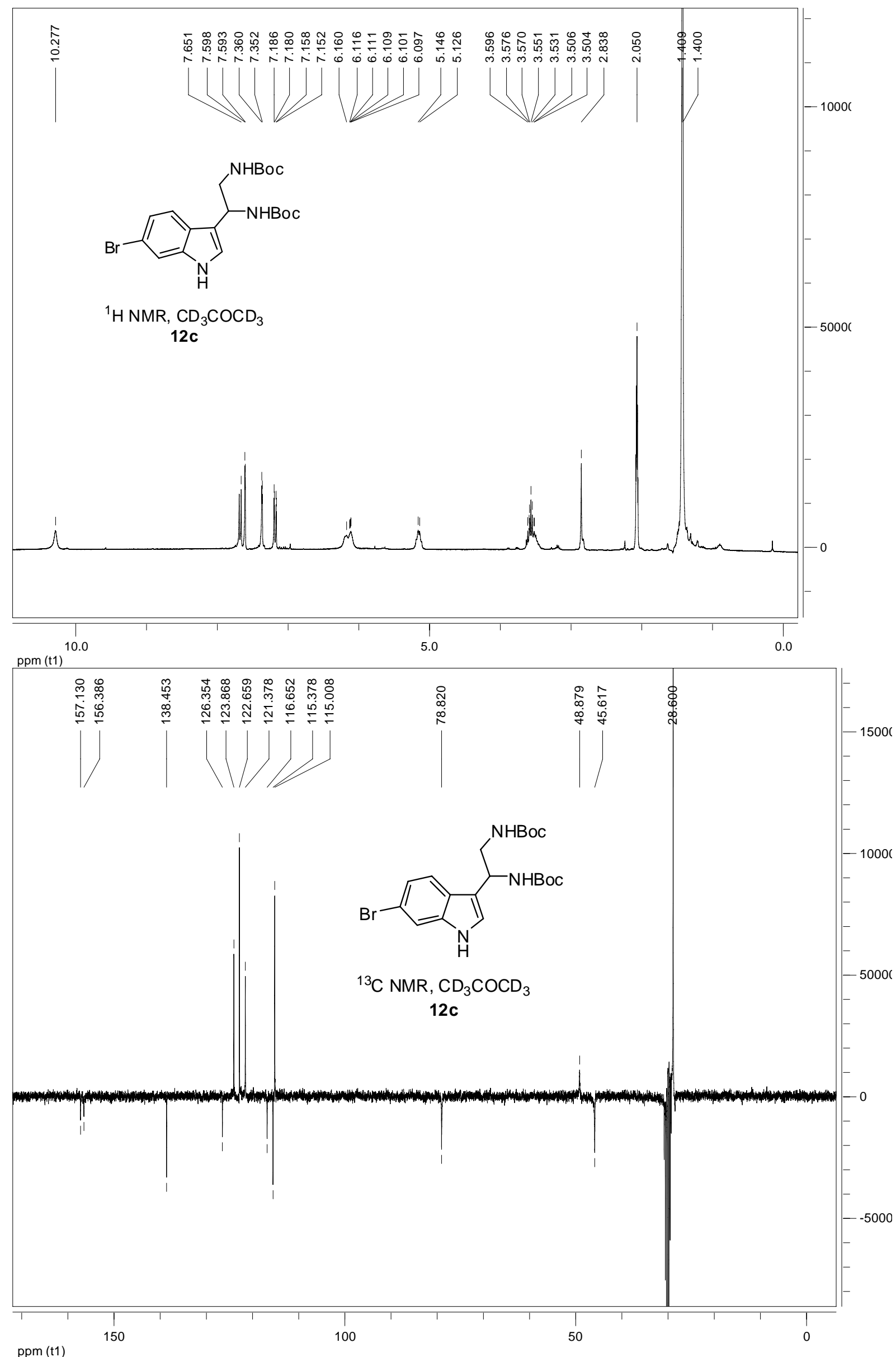